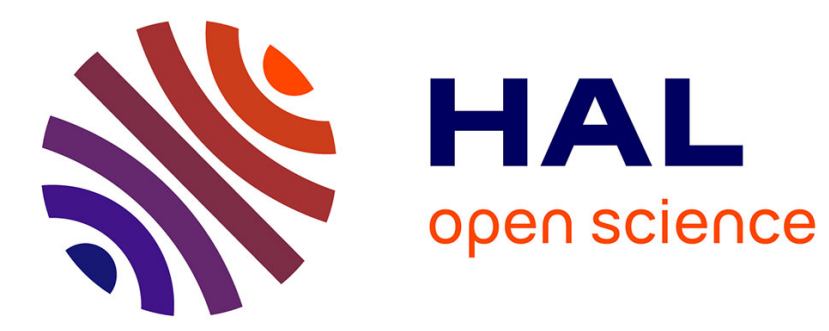

\title{
Triple-line kinetics for solid films
}

Ashwani K. Tripathi, Olivier Pierre-Louis

\section{To cite this version:}

Ashwani K. Tripathi, Olivier Pierre-Louis. Triple-line kinetics for solid films. Physical Review E , 2018, 97 (2), pp.022801. 10.1103/PhysRevE.97.022801 . hal-02289856

\section{HAL Id: hal-02289856 \\ https://univ-lyon1.hal.science/hal-02289856}

Submitted on 11 Feb 2021

HAL is a multi-disciplinary open access archive for the deposit and dissemination of scientific research documents, whether they are published or not. The documents may come from teaching and research institutions in France or abroad, or from public or private research centers.
L'archive ouverte pluridisciplinaire HAL, est destinée au dépôt et à la diffusion de documents scientifiques de niveau recherche, publiés ou non, émanant des établissements d'enseignement et de recherche français ou étrangers, des laboratoires publics ou privés. 


\title{
Triple-line kinetics for solid films
}

\author{
Ashwani K. Tripathi and Olivier Pierre-Louis* \\ Institut Lumière Matière, UMR No. 5306 Université Lyon 1, CNRS, Université de Lyon, 69622 Villeurbanne, France
}

(Received 29 September 2017; published 12 February 2018)

\begin{abstract}
We present a derivation of the triple-line kinetic boundary conditions for a solid film in contact with a solid substrate for both nonconserved (evaporation-condensation) and conserved (surface diffusion) dynamics. The result is obtained via a matched asymptotic expansion from a mesoscopic model with a thickness-dependent wetting potential (or disjoining pressure) and mobility. In the nonconserved case, we obtain a single boundary condition, which relates the triple-line velocity with the deviation of the contact angle from its equilibrium value. In the conserved case, two kinetic boundary conditions are needed. They relate the velocity and mass flux at the triple line to the contact angle deviation and discontinuity of the chemical potential. These linear relations are described by three kinetic coefficients. The conditions under which the kinetic coefficients remain finite are obtained. We find, for example, that some kinetic coefficients diverge within the conserved model in the presence of van der Waals interaction.
\end{abstract}

DOI: 10.1103/PhysRevE.97.022801

\section{INTRODUCTION}

Wetting concepts play a key role in the understanding of the morphologies of thin solid films emerging from crystal growth or from solid-state dewetting. One central result in liquid wetting is the Young-Dupré equilibrium contact angle condition at the triple line

$$
\bar{\gamma} \cos \theta_{\mathrm{eq}}=\gamma_{\mathrm{SV}}-\gamma_{\mathrm{SF}},
$$

where $\gamma_{\mathrm{SV}}, \gamma_{\mathrm{SF}}$, and $\bar{\gamma}$ are the surface energy densities of substrate-vapor, substrate-film, and film-vapor interfaces, respectively. The nonequilibrium deviation from this relation has been a central issue in the study of liquid spreading and dewetting $[1,2]$. Indeed, this question appears to be critical for hydrodynamic theories due to the divergence of viscous dissipation at the triple line [3], which leads to a large contribution to dissipation localized at the triple line (this divergence must be cured by some microscopic cutoff in order to allow for tripleline motion). In order to account for this triple-line dissipation, a deviation from the equilibrium Young-Dupré equation (1) with a kinetic coefficient $k$ has been proposed [1,2],

$$
v=k\left(\cos \theta_{\mathrm{eq}}-\cos \theta\right),
$$

where $v$ is the triple-line velocity and $\theta$ is the actual contact angle. It is therefore natural to ask if similar deviations from the equilibrium contact angle condition may be observed for solid films. Indeed, experimental studies have reported deviations from the equilibrium contact angle not only in liquid films [1,4-7], but also at triple junctions between grains in polycrystalline materials $[8,9]$, a system which is closer to thin solid films. Our goal in this paper is to derive kinetic boundary conditions which describe the nonequilibrium behavior of the triple line for solid-state thin films.

Several modeling approaches have already been developed to study morphological changes in solid films, such as Mullins'

*Corresponding author: olivier.pierre-louis@univ-lyon1.fr continuum models for evaporation-condensation or surface diffusion [10,11], a strongly anisotropic surface diffusion model [12], phase field models [13], or kinetic Monte Carlo models $[14,15]$. Continuum models usually assume a fixed contact angle at the triple line, except for that in Ref. [16], where a nonequilibrium relation similar to Eq. (2) was proposed, allowing for the relaxation of the triple-line contact angle. This condition improves the stability of numerical modeling of solid-state dewetting. However, the physical relevance or microscopic interpretation of the related mobility remains an open question.

Our analysis is based on a mesoscopic model generalizing Mullins' model [17] with a thickness-dependent free-energy density. The thickness-dependent part of the free energy is called the wetting potential and its derivative with respect to the thickness is the disjoining pressure. Similar models have also been used in the case of liquid films [1,2,18-21]. The profile of the wetting potential can be tuned so as to obtain complete wetting or partial wetting [1,22]. For example, solid films exhibiting complete wetting correspond to a monotonically decreasing wetting potential. Such a wetting potential has been used to enforce the presence of a continuous wetting layer in the case of unstable films with a destabilization originating from faceting instabilities [23] or heteroepitaxial elastic strain [24]. The case of partial wetting, where the wetting potential exhibits a negative minimum, has also been studied in solid films to describe the formation of holes [25] or Ostwald ripening of solid nanoislands [26].

Our strategy is to start with a generalized mesoscopic model including not only a thickness-dependent free energy, but also a thickness-dependent mobility. Using this model, we perform an asymptotic matched expansion assuming a separation of scales between the scale of the triple-line region, where the wetting potential and mobility vary, and a larger scale related to the variations of film and substrate profiles. The expansion procedure is similar to that used to obtain boundary conditions in phase field models [27] and especially in the presence of space-dependent kinetics in the interface region [28]. 
We consider both nonconserved (evaporationcondensation) and conserved (surface diffusion) dynamics. In the nonconserved case, our results lead to a boundary condition similar to Eq. (2) with one kinetic coefficient describing the deviation of the contact angle. However, there are two boundary conditions in the conserved case, involving the departure of the contact angle from its equilibrium value and the mass flux through the triple line. This leads to three kinetic coefficients.

We obtain explicit expressions for the kinetic coefficients both in the nonconserved and in the conserved cases. These expressions provide strong conditions on the convergence of the wetting potential and mobility to their asymptotic values for large thicknesses in order to ensure the finiteness of the kinetic coefficients. These conditions are not necessarily satisfied in all physical systems. For example, in the conserved case, some kinetic coefficients diverge when the dominant contribution to the wetting potential is the usual van der Waals interaction.

We use the example of solid-state dewetting dynamics from a straight film edge to check quantitatively our results. This process occurs when a thin film is heated below melting to a sufficiently high temperature where either evaporation or surface diffusion is activated [10,29,30]. Surface-diffusionlimited dewetting has been observed in many experiments such as $\mathrm{Si} / \mathrm{SiO}_{2}$ [31-36], Ni/MgO [12,29,37], Au on fused silica [38], or $\mathrm{Cu}$ on sapphire [39].

The paper is organized as follows. In Sec. II, we discuss the equilibrium morphology of the film and the corresponding boundary condition at the triple line. In Sec. III, we present the nonconserved model and derive the corresponding tripleline kinetic boundary condition. In Sec. IV, we perform a similar analysis starting from the conserved model (the details of the derivation in this case are reported in Appendix A). Section $\mathrm{V}$ discusses the physical implications of our results. We summarize the results in Sec. VI.

\section{SURFACE FREE ENERGY AND EQUILIBRIUM SHAPE}

We consider a one-dimensional model with a surface free energy which is isotropic but which depends upon the thickness $h$ of the film

$$
\mathcal{F}=\int d s \gamma(h)
$$

where $\gamma(h)$ is the surface energy density of the film and $d s=$ $\left[1+\left(\partial_{x} h\right)^{2}\right]^{1 / 2} d x$ is the surface arc-length element. We rewrite the surface free energy as

$$
\gamma(h)=\bar{\gamma}+w(h)
$$

where $\bar{\gamma}$ is the asymptotic surface energy of the film for large $h$, so that $w(h) \rightarrow 0$ as $h \rightarrow \infty$. The deviation from this asymptotic value, denoted by $w(h)$ and called the wetting potential, arises due to various physical effects such as breaking of interatomic bonds extending farther than nearest neighbors, structural effects, electrostatic effects, and van der Waals interactions $[22,40,41]$. Here we consider that the substrate corresponds to a well-defined stable state. This state can correspond to a wetting film or to a bare substrate with an adsorbate layer, as discussed, e.g., in Ref. [26]. The variation of $w(h)$ with film thickness is shown in Fig. 1.

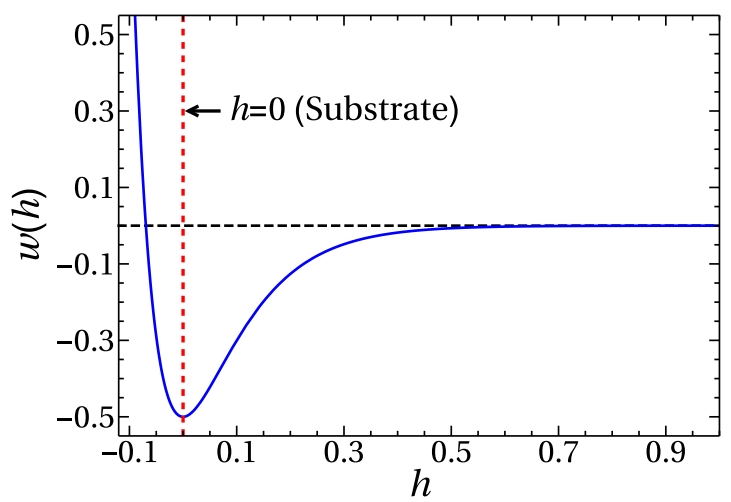

FIG. 1. Wetting potential as a function of film thickness in the case of partial wetting. By convention, the origin of heights $(h=0)$ corresponds to the substrate equilibrium height at the minimum of $w$.

Under the constraint that the total number of particles $\mathcal{N}$ in the film is conserved, with

$$
\mathcal{N}=\frac{1}{\Omega} \int d x h,
$$

the equilibrium shape of the film is obtained from the minimization of the Gibbs free energy $\mathcal{G}=\mathcal{F}-\mu_{\text {eq }} \mathcal{N}$, where $\mu_{\text {eq }}$ is the chemical potential of the film and $\Omega$ is the atomic volume. This leads to the equilibrium equation $\mu_{\mathrm{eq}}=\mu$, where $\mu_{\mathrm{eq}}$ is a constant and

$$
\frac{\mu}{\Omega}=\gamma(h) \kappa+\frac{\gamma^{\prime}(h)}{\left[1+\left(\partial_{x} h\right)^{2}\right]^{1 / 2}},
$$

where $\gamma^{\prime}(h)$ denotes the derivative of $\gamma(h)$ with respect to $h$ and

$$
\kappa=-\frac{\partial_{x x} h}{\left[1+\left(\partial_{x} h\right)^{2}\right]^{3 / 2}}
$$

is the surface curvature.

On the substrate side far from the triple line, the height is constant and equal to $h_{\text {sub }}$. Thus, Eq. (6) leads to $\mu_{\text {eq }}=$ $\Omega \gamma^{\prime}\left(h_{\text {sub }}\right)$. In addition, far from the substrate on the side where the film is thick, we have $\gamma(h) \approx \bar{\gamma}$, so Eq. (6) reads $\mu_{\text {eq }}=$ $\Omega \bar{\gamma} \kappa$. Thus, the equilibrium profile far from the substrate is an arc of a circle with a radius $R=1 / \kappa=\Omega \bar{\gamma} / \mu_{\text {eq }}$ obeying

$$
h_{\text {isl }}(x)=h_{m}+\left(R^{2}-x^{2}\right)^{1 / 2}-R,
$$

where $h_{m}$ is the height at the apex of the island. Furthermore, rewriting Eq. (6) as

$$
\partial_{x}\left(\frac{\gamma(h)}{\left[1+\left(\partial_{x} h\right)^{2}\right]^{1 / 2}}\right)=\frac{\mu_{\mathrm{eq}}}{\Omega} \partial_{x} h
$$

and integrating along $x$, we obtain the condition of balance of the film tensions [1,22]

$$
\gamma\left(h_{\mathrm{sub}}\right)-\frac{\mu_{\mathrm{eq}}}{\Omega} h_{\mathrm{sub}}=\frac{\gamma(h)}{\left[1+\left(\partial_{x} h\right)^{2}\right]^{1 / 2}}-\frac{\mu_{\mathrm{eq}}}{\Omega} h .
$$

Using this condition at the top of the island, we find an equation that determines $h_{m}$,

$$
\gamma\left(h_{\mathrm{sub}}\right)-\frac{\mu_{\mathrm{eq}}}{\Omega} h_{\mathrm{sub}}=\bar{\gamma}-\frac{\mu_{\mathrm{eq}}}{\Omega} h_{m} .
$$


We now wish to write the boundary condition at the triple line. We choose by convention to define the triple-line position as $x_{\mathrm{TL}}$. The location of $x_{\mathrm{TL}}$ is a priori arbitrary, but should be in the triple-line region. We also define $\theta_{\mathrm{TL}}$ as the extrapolation of the orientation angle $\theta=\arctan \left(\partial_{x} h\right)$ of the macroscopic equilibrium island profile at the triple line: $\theta_{\mathrm{TL}}^{\mathrm{eq}}=\arctan \left[\partial_{x} h_{\mathrm{isl}}\left(x_{\mathrm{TL}}\right)\right]$. Combining the relation $\cos \theta=\left(R^{2}-x^{2}\right)^{1 / 2} / R$ in the island profile with the previous expressions, we obtain

$$
\bar{\gamma} \cos \theta_{\mathrm{TL}}^{\mathrm{eq}}-\gamma\left(h_{\mathrm{sub}}\right)=\frac{\mu_{\mathrm{eq}}}{\Omega}[h]_{\mathrm{TL}},
$$

where $[h]_{\mathrm{TL}}=h_{\text {isl }}\left(x_{\mathrm{TL}}\right)-h_{\text {sub }}$ is the possible discontinuity in the film thickness when approaching the triple line from the island and substrate sides with the macroscopic profiles.

If we prescribe a definition of the triple-line position such that the discontinuity $[h]_{\mathrm{TL}}$ vanishes, then from Eq. (12) we obtain the contact angle relation

$$
\bar{\gamma} \cos \theta_{\mathrm{TL}}^{\mathrm{eq}}=\gamma\left(h_{\mathrm{sub}}\right)=\bar{\gamma}+w\left(h_{\mathrm{sub}}\right) .
$$

If in addition the radius $R$ of the island is large, then the chemical potential $\mu_{\mathrm{eq}}=\Omega \bar{\gamma} / R$ is small. Since $\mu_{\mathrm{eq}}=\Omega \gamma^{\prime}\left(h_{\mathrm{sub}}\right)$, we also expect that $\gamma^{\prime}\left(h_{\text {sub }}\right)=0$, i.e., that the substrate height is at the minimum of the wetting potential. By convention, this minimum is at $h=0$, as shown in Fig. 1, and we obtain the Young-Dupré law for large islands

$$
\bar{\gamma} \cos \theta_{\mathrm{eq}}=\gamma(0)=\bar{\gamma}+w(0) .
$$

Comparison with Eq. (1) therefore imposes that $w(0)=\gamma_{\mathrm{SV}}-$ $\gamma_{\mathrm{SF}}-\bar{\gamma}$, i.e., $w(0)$ is equal to the usual spreading coefficient [1]. When the wetting potential exhibits a minimum of moderate depth $0>w(0)>-2 \bar{\gamma}$, then Eq. (14) indicates that there is a well-defined contact angle $0<\theta_{\text {eq }}^{\infty}<\pi$. This situation, called partial wetting, will be assumed in the following.

\section{EVAPORATION-CONDENSATION DYNAMICS}

\section{A. Model equations}

The first dynamical model describes evaporation or the growth process of a solid film in contact with a threedimensional vapor phase. Following Mullins [17], the normal velocity $v_{n}$ of the film profile is given by

$$
v_{n}=m\left(\frac{\mu_{c}}{\Omega}-\frac{\mu}{\Omega}\right),
$$

where $m$ is the transport coefficient or mobility, $\mu_{c}$ is the chemical potential of the vapor phase, and $\mu$ is the spacedependent local chemical potential of the film, given by Eq. (6). The rate of change of film thickness $\partial_{t} h$ is related to $v_{n}$ as

$$
v_{n}=\frac{\partial_{t} h}{\left[1+\left(\partial_{x} h\right)^{2}\right]^{1 / 2}} .
$$

For the sake of simplicity, we consider the small slope limit $\partial_{x} h \ll 1$, or $\theta \ll 1$. Then, as a consequence of Eq. (14), we also have $w(h) \ll \bar{\gamma}$. Therefore, Eq. (15) now reads

$$
\partial_{t} h=m(h)\left(\frac{\mu_{c}}{\Omega}+\bar{\gamma} \partial_{x}^{2} h-w^{\prime}(h)\right),
$$

where $m(h)$ indicates explicitly the dependence of the mobility on the film thickness.

\section{B. Kinetic boundary conditions \\ 1. Matched asymptotic expansion}

In this section, we obtain a kinetic boundary condition starting from Eq. (17). In order to perform the asymptotic expansion, we define a small length scale $\epsilon$ which is of the order of the range of the wetting potential $w(h)$ and the mobility $m(h)$ and small as compared to the typical thickness of the film $\bar{h}$. The dependence of $w(h)$ and $m(h)$ on $\epsilon$ is made explicit via

$$
w(h)=W(h / \epsilon), \quad m(h)=M(h / \epsilon),
$$

where $W$ and $M$ do not depend on $\epsilon$. In the limit of large thicknesses $h \gg \epsilon$, we assume that $W(h / \epsilon) \rightarrow W(\infty)=0$ and $M(h / \epsilon) \rightarrow M(\infty)$, where $M(\infty)$ is a constant (we also assume that all the derivatives of $W$ and $M$ vanish as $h \gg \epsilon$ for definiteness, indicating that $W$ and $M$ are smooth regular functions). Using Eq. (18), we rewrite Eq. (17) in a way which makes the dependence on $\epsilon$ explicit:

$$
\partial_{t} h=M(h / \epsilon)\left(\frac{\mu_{c}}{\Omega}+\bar{\gamma} \partial_{x}^{2} h-\frac{1}{\epsilon} W^{\prime}(h / \epsilon)\right) .
$$

In order to obtain an effective boundary condition by means of an asymptotic matched expansion, we divide the full film surface into three regions.

(a) Island region. This corresponds to the region of film where the effect of the wetting potential is negligible. We expect that the length-scale of variation in this region is of $O\left(\epsilon^{0}\right)$, i.e.,

$$
x \sim O\left(\epsilon^{0}\right), \quad h \sim O\left(\epsilon^{0}\right) .
$$

We also make the dependence of $h$ on $\epsilon$ explicit by using the notation $h(x, t ; \epsilon)$.

(b) Substrate region. This is the region far from the triple line where there is no island. We define the following scaling ansatz in this region:

$$
x=\chi, \quad h(x, t ; \epsilon)=\epsilon \mathcal{H}(\chi, t ; \epsilon) .
$$

(c) Triple-line (TL) region. This corresponds to the region between the film and the substrate. The length scale of variation in this region is set by $\epsilon$, suggesting the following ansatz:

$$
x=x_{\mathrm{TL}}+\epsilon X, \quad h(x, t ; \epsilon)=\epsilon H(X, t ; \epsilon) .
$$

As an important remark, we note that these definitions correspond to a reference frame with a vanishing triple-line position $X_{\mathrm{TL}}=0$ in the TL region.

A schematic picture of these three regions is shown in Fig. 2. In the three regions, Eq. (19) can be written as

$$
\begin{gathered}
\partial_{t} h-v \partial_{x} h=M(\infty)\left[\frac{\mu_{c}}{\Omega}+\bar{\gamma} \partial_{x}^{2} h(x, t)\right], \\
\epsilon^{2} \partial_{t} H-\epsilon v \partial_{X} H=M(H)\left[\epsilon \frac{\mu_{c}}{\Omega}+\bar{\gamma} \partial_{X}^{2} H-W^{\prime}(H)\right], \\
\epsilon^{2} \partial_{t} \mathcal{H}-\epsilon^{2} v \partial_{\chi} \mathcal{H}=M(\mathcal{H})\left[\epsilon \frac{\mu_{c}}{\Omega}+\bar{\gamma} \epsilon^{2} \partial_{\chi}^{2} \mathcal{H}-W^{\prime}(\mathcal{H})\right],
\end{gathered}
$$

where equations are written in the coordinate system comoving with the triple line with a velocity $v=\dot{x}_{\mathrm{TL}}$. Furthermore, the matching condition between the triple-line and island regions is given by

$$
h\left(x_{\mathrm{TL}}+\epsilon X, t ; \epsilon\right)=\epsilon H(X, t ; \epsilon)
$$




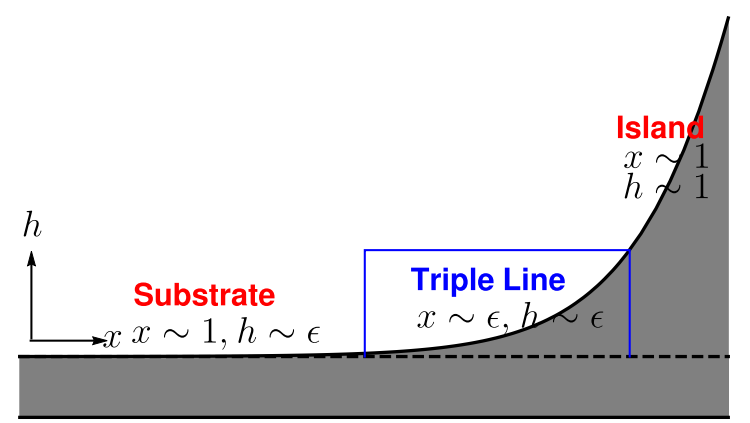

FIG. 2. Schematic showing the division of the full film profile into substrate, triple-line, and island regions. The dashed line indicates the equilibrium substrate height.

as $X \rightarrow+\infty$ and $\epsilon \rightarrow 0$ with $\epsilon X \rightarrow 0^{+}$. Similarly, the matching condition between the substrate and TL regions is given by

$$
H(X, t ; \epsilon)=\mathcal{H}\left(\chi_{\mathrm{TL}}+\epsilon X, t ; \epsilon\right)
$$

as $x \rightarrow-\infty, \epsilon \rightarrow 0$, and $\epsilon X \rightarrow 0^{-}$.

We expand the film profile in the three regions as follows:

$$
\begin{gathered}
h(x, t ; \epsilon)=h_{0}(x, t)+\epsilon h_{1}(x, t)+\epsilon^{2} h_{2}(x, t)+\cdots, \\
H(X, t ; \epsilon)=H_{0}(X, t)+\epsilon H_{1}(X, t)+\epsilon^{2} H_{2}(X, t)+\cdots, \\
\mathcal{H}(\chi, t ; \epsilon)=\mathcal{H}_{0}(\chi, t)+\epsilon \mathcal{H}_{1}(\chi, t)+\epsilon^{2} \mathcal{H}_{2}(\chi, t)+\cdots .
\end{gathered}
$$

We also formally expand the triple-line velocity as

$$
v=v_{0}+\epsilon v_{1}+\epsilon^{2} v_{2}+\cdots .
$$

The matching condition between the TL and island regions [Eq. (26)] is then expanded using Eqs. (28) and (29). Up to third order, the expanded matching conditions are given by

$$
\begin{aligned}
& h_{0}\left(x_{\mathrm{TL}}\right)=0 \text {, } \\
& H_{0}(X, t)=X \partial_{x} h_{0}\left(x_{\mathrm{TL}}\right)+h_{1}\left(x_{\mathrm{TL}}\right), \\
& H_{1}(X, t)=\frac{X^{2}}{2} \partial_{x}^{2} h_{0}\left(x_{\mathrm{TL}}\right) \\
& +X \partial_{x} h_{1}\left(x_{\mathrm{TL}}\right)+h_{2}\left(x_{\mathrm{TL}}\right), \\
& H_{2}(X, t)=\frac{X^{3}}{6} \partial_{x}^{3} h_{0}\left(x_{\mathrm{TL}}\right)+\frac{X^{2}}{2} \partial_{x}^{2} h_{1}\left(x_{\mathrm{TL}}\right) \\
& +X \partial_{x} h_{2}\left(x_{\mathrm{TL}}\right)+h_{3}\left(x_{\mathrm{TL}}\right), \\
& H_{3}(X, t)=\frac{X^{4}}{24} \partial_{x}^{4} h_{0}\left(x_{\mathrm{TL}}\right)+\frac{X^{3}}{6} \partial_{x}^{3} h_{1}\left(x_{\mathrm{TL}}\right) \\
& +\frac{X^{2}}{2} \partial_{x}^{2} h_{2}\left(x_{\mathrm{TL}}\right)+X \partial_{x} h_{3}\left(x_{\mathrm{TL}}\right) \\
& +h_{4}\left(x_{\mathrm{TL}}\right) \text {. }
\end{aligned}
$$

Similarly, the matching condition between the TL and substrate regions [Eq. (27)] is expanded as

$$
\begin{gathered}
H_{0}(X, t)=\mathcal{H}_{0}\left(\chi_{\mathrm{TL}}\right), \\
H_{1}(X, t)=X \partial_{\chi} \mathcal{H}_{0}\left(\chi_{\mathrm{TL}}\right)+\mathcal{H}_{1}\left(\chi_{\mathrm{TL}}\right),
\end{gathered}
$$

$$
\begin{aligned}
H_{2}(X, t)= & \frac{X^{2}}{2} \partial_{\chi}^{2} \mathcal{H}_{0}\left(\chi_{\mathrm{TL}}\right)+X \partial_{\chi} \mathcal{H}_{1}\left(\chi_{\mathrm{TL}}\right) \\
& +\mathcal{H}_{2}\left(\chi_{\mathrm{TL}}\right), \\
H_{3}(X, t)= & \frac{X^{3}}{6} \partial_{\chi}^{3} \mathcal{H}_{0}\left(\chi_{\mathrm{TL}}\right)+\frac{X^{2}}{2} \partial_{\chi}^{2} \mathcal{H}_{1}\left(\chi_{\mathrm{TL}}\right) \\
& +X \partial_{\chi} \mathcal{H}_{2}\left(\chi_{\mathrm{TL}}\right)+\mathcal{H}_{3}\left(\chi_{\mathrm{TL}}\right) .
\end{aligned}
$$

\section{Far-field behavior}

The evolution equation in the film region [Eq. (23)] does not depend explicitly on $\epsilon$. As a consequence, it is not affected by the expansion. However, there is an explicit dependence on $\epsilon$ for the far-field evolution equation in the substrate region [Eq. (25)]. We therefore expand this equation using Eq. (30). To zeroth order we obtain

$$
M\left(\mathcal{H}_{0}\right) W^{\prime}\left(\mathcal{H}_{0}\right)=0 .
$$

Assuming that the mobility is nonzero, and since the minimum of $W$ is located at $\mathcal{H}=0$, this relation implies that $\mathcal{H}_{0}=0$. Similarly to first order in $\epsilon$, we find

$$
M\left(\mathcal{H}_{0}\right)\left[\frac{\mu_{c}}{\Omega}-W^{\prime \prime}\left(\mathcal{H}_{0}\right) \mathcal{H}_{1}\right]=0,
$$

which gives the first-order contribution to the substrate profile

$$
\mathcal{H}_{1}=\frac{\mu_{c}}{\Omega W^{\prime \prime}(0)} .
$$

Note that the dynamics of the substrate profile appears only to higher order in $\epsilon$, as seen from the prefactor $\epsilon^{2}$ of the terms on the left-hand side of Eq. (25).

\section{The TL region: Preamble to the expansion}

We now focus on the TL region. Before performing the order by order expansion of the profile in the TL region, we write down an additional relation that will be useful in the following. For this purpose, we multiply Eq. (24) by $\partial_{X} H$ and integrate, leading to

$$
\begin{aligned}
& \epsilon^{2} \int_{X_{-}}^{X_{+}} \frac{\partial_{t} H \partial_{X} H}{M(H)} d X-\epsilon v \int_{X_{-}}^{X_{+}} \frac{\left(\partial_{X} H\right)^{2}}{M(H)} d X \\
& \quad=\epsilon \frac{\mu_{c}}{\Omega}\left[H\left(X_{+}\right)-H\left(X_{-}\right)\right]-\left[U\left(X_{+}\right)-U\left(X_{-}\right)\right],
\end{aligned}
$$

where $X_{ \pm}$are two points in the TL region. In addition, we have defined the normalized quantity

$$
U(X)=-\frac{\bar{\gamma}}{2}\left(\partial_{X} H\right)^{2}+W(H),
$$

which corresponds in physical coordinates to

$$
u(x)=-\frac{\bar{\gamma}}{2}\left(\partial_{x} h\right)^{2}+w(h) .
$$

\section{Zeroth order in the TL region}

To zeroth order, Eq. (24) leads to

$$
M\left(H_{0}\right)\left[\bar{\gamma} \partial_{X}^{2} H_{0}-W^{\prime}\left(H_{0}\right)\right]=0 .
$$

In the limit $X \rightarrow-\infty$, using the matching condition (37), we obtain

$$
W^{\prime}\left[\mathcal{H}_{0}\left(\chi_{\mathrm{TL}}\right)\right]=0,
$$


which implies that $\mathcal{H}_{0}\left(\chi_{\mathrm{TL}}\right)=0$. This is consistent with the far-field behavior on the substrate, as discussed above. To this order, Eq. (44) reads

$$
U_{0}\left(X_{+}\right)-U_{0}\left(X_{-}\right)=0
$$

with

$$
U_{0}(X)=-\frac{\bar{\gamma}}{2}\left(\partial_{X} H_{0}\right)^{2}+W\left(H_{0}\right)
$$

Taking the limits $X_{+} \rightarrow+\infty$ and $X_{-} \rightarrow-\infty$ and using the matching conditions (33) and (37), we obtain

$$
\frac{\bar{\gamma}}{2}\left[h_{0}^{\prime}\left(x_{\mathrm{TL}}\right)\right]^{2}=W(\infty)-W(0),
$$

where $W(\infty)=0$, by definition, and $h_{0}^{\prime}\left(x_{\mathrm{TL}}\right)=\partial_{x} h_{0}\left(x_{\mathrm{TL}}\right)$ defines the slope of the film profile at the triple line. We will use similar notation for the other derivatives in the following, such as $h_{0}^{\prime \prime}\left(x_{\mathrm{TL}}\right)=\partial_{x}^{2} h_{0}\left(x_{\mathrm{TL}}\right)$. Within the small slope limit,

$$
\frac{1}{2}\left[h_{0}^{\prime}\left(x_{\mathrm{TL}}\right)\right]^{2}=\frac{1}{2} \tan ^{2} \theta_{\mathrm{eq}} \approx \frac{1}{2} \theta_{\mathrm{eq}}^{2} \approx 1-\cos \theta_{\mathrm{eq}} .
$$

Therefore, Eq. (51) is equivalent to the Young-Dupré equations (1) and (14). As a summary to zeroth order, the contact line is stationary and the contact angle is equal to its equilibrium value.

\section{First order in the TL region}

The first-order contribution to Eq. (24) is

$$
\begin{aligned}
-v_{0} \partial_{X} H_{0}= & M\left(H_{0}\right)\left[\bar{\gamma} \partial_{X}^{2} H_{1}-W^{\prime \prime}\left(H_{0}\right) H_{1}+\frac{\mu_{c}}{\Omega}\right] \\
& +M^{\prime}\left(H_{0}\right) H_{1}\left[\bar{\gamma} \partial_{X}^{2} H_{0}-W^{\prime}\left(H_{0}\right)\right] .
\end{aligned}
$$

From Eq. (47), the last term in Eq. (53) vanishes. In the limit $X \rightarrow+\infty$, using the matching conditions (33) and (34) and the relation $W^{\prime \prime}(\infty)=0$, we find

$$
-\frac{v_{0} h_{0}^{\prime}\left(x_{\mathrm{TL}}\right)}{M(\infty)}=-\frac{\mu_{1}(\infty)}{\Omega}+\frac{\mu_{c}}{\Omega} .
$$

The first term on the right-hand side is the first-order contribution to the chemical potential in the island region, $\mu_{1}(\infty)=$ $-\Omega \bar{\gamma} h_{0}^{\prime \prime}\left(x_{\mathrm{TL}}\right)$. Similarly, the $X \rightarrow-\infty$ limit leads to

$$
\frac{\mu_{c}}{\Omega}-W^{\prime \prime}(0)\left[X \mathcal{H}_{0}^{\prime}\left(\chi_{\mathrm{TL}}\right)+\mathcal{H}_{1}\left(\chi_{\mathrm{TL}}\right)\right]=0 .
$$

Both the constant term and the diverging term must vanish. As a consequence, $\mathcal{H}_{0}^{\prime}\left(\chi_{\mathrm{TL}}\right)=0$ and we find $\mu_{c}=\mu_{1}(-\infty)=$ $\Omega W^{\prime \prime}(0) \mathcal{H}_{1}\left(\chi_{\mathrm{TL}}\right)$, which is consistent with Eq. (43).

The kinetic boundary condition arises from Eq. (44) to first order

$$
\begin{aligned}
-v_{0} \int_{X_{-}}^{X_{+}} \frac{\left(\partial_{X} H_{0}\right)^{2}}{M\left(H_{0}\right)} d X= & \frac{\mu_{c}}{\Omega}\left[H_{0}\left(X_{+}\right)-H_{0}\left(X_{-}\right)\right] \\
& -\left[U_{1}\left(X_{+}\right)-U_{1}\left(X_{-}\right)\right]
\end{aligned}
$$

where $U_{1}(X)$ is given by

$$
U_{1}(X)=-\bar{\gamma}\left(\partial_{X} H_{0}\right)\left(\partial_{X} H_{1}\right)+W^{\prime}\left(H_{0}\right) H_{1} .
$$

In the limits $X_{+} \rightarrow+\infty$ and $X_{-} \rightarrow-\infty$, we obtain

$$
v_{0} \int_{-\infty}^{+\infty} \frac{\left(\partial_{X} H_{0}\right)^{2}}{M\left(H_{0}\right)} d X=\left[U_{1}\right]_{-\infty}^{+\infty}-\frac{\mu_{c}}{\Omega}\left[H_{0}\right]_{-\infty}^{+\infty},
$$

where $[A]_{-\infty}^{+\infty}$ denotes the difference of $A$ calculated in island and substrate regions. At this stage, Eq. (58) involves diverging contributions, both on the left-hand side and on the right-hand side However, these diverging contributions cancel.

In order to see how these cancellations occur, we write the diverging contributions explicitly. Using the matching conditions in the island and substrate regions, we have

$$
\left[U_{1}\right]_{-\infty}^{+\infty}=-\bar{\gamma} X_{+} h_{0}^{\prime}\left(x_{\mathrm{TL}}\right) h_{0}^{\prime \prime}\left(x_{\mathrm{TL}}\right)+\left[\tilde{U}_{1}\right]_{-\infty}^{+\infty},
$$

with $X_{+} \rightarrow+\infty$, where

$$
\left[\tilde{U}_{1}\right]_{-\infty}^{+\infty}=-\bar{\gamma} h_{0}^{\prime}\left(x_{\mathrm{TL}}\right) h_{1}^{\prime}\left(x_{\mathrm{TL}}\right)
$$

represents the nondiverging part of $\left[U_{1}\right]$. Similarly,

$$
\frac{\mu_{c}}{\Omega}\left[H_{0}\right]_{-\infty}^{+\infty}=\frac{\mu_{c}}{\Omega} X_{+} h_{0}^{\prime}\left(x_{\mathrm{TL}}\right)+\frac{\mu_{c}}{\Omega}\left[\tilde{H}_{0}\right]_{-\infty}^{+\infty} .
$$

We also rewrite the left-hand side of Eq. (58) as

$$
\begin{aligned}
v_{0} \int_{-\infty}^{+\infty} \frac{\left(\partial_{X} H_{0}\right)^{2}}{M\left(H_{0}\right)} d X \\
=v_{0} \int_{-\infty}^{+\infty}\left[\frac{\left(\partial_{X} H_{0}\right)^{2}}{M\left(H_{0}\right)}-\Theta(X) \frac{\left[h_{0}^{\prime}\left(x_{\mathrm{TL}}\right)\right]^{2}}{M(\infty)}\right] d X \\
\quad+\frac{v_{0} X_{+}\left[h_{0}^{\prime}\left(x_{\mathrm{TL}}\right)\right]^{2}}{M(\infty)},
\end{aligned}
$$

where we have defined the Heaviside function $\Theta(X)=1$ when $X>0$, and $\Theta(X)=0$ otherwise. Using Eq. (54), we see that the diverging term proportional to $X_{+}$in Eq. (62) cancels with those coming from Eqs. (59) and (61), and finally Eq. (58) leads to

$$
v_{0} L_{v}=\left[\tilde{U}_{1}\right]_{-\infty}^{+\infty}-\frac{\mu_{c}}{\Omega}\left[\tilde{H}_{0}\right]_{-\infty}^{+\infty},
$$

where the kinetic coefficient $L_{v}$ is given by

$$
L_{v}=\int_{-\infty}^{+\infty}\left[\frac{\left(\partial_{X} H_{0}\right)^{2}}{M\left(H_{0}\right)}-\Theta(X) \frac{\left[h_{0}^{\prime}\left(x_{\mathrm{TL}}\right)\right]^{2}}{M(\infty)}\right] d X .
$$

\section{The TL boundary condition}

Combining the zeroth- and the first-order contributions, viz., Eqs. (49) and (63), we obtain the kinetic boundary condition up to first order

$$
v \mathcal{L}_{v}=[u]_{\mathrm{TL}}-\mu_{c}[h]_{\mathrm{TL}},
$$

where

$$
\mathcal{L}_{v}=\epsilon L_{v} .
$$

In addition, the discontinuity of any quantity $y$ at the triple line extrapolated from the far-field profiles and in physical variables is denoted by $[y]_{\mathrm{TL}}$. Here, for example,

$$
[u]_{\mathrm{TL}}=[\tilde{U}]_{-\infty}^{+\infty}=\left[\tilde{U}_{0}\right]_{-\infty}^{+\infty}+\epsilon\left[\tilde{U}_{1}\right]_{-\infty}^{+\infty}+O\left(\epsilon^{2}\right) .
$$

The triple-line position is arbitrary in our expansion; it can be placed anywhere in the TL region. Let us also recall that, by convention, we have defined the location of the triple line at $X=0$ in the TL region. Since the contact angle is fixed at equilibrium, it is tempting to use the surface slope as an effective order parameter reaching asymptotically constant values in the substrate and island regions at equilibrium. Using this analogy, we define the triple-line position via a Gibbs 
dividing surfacelike criterion. Using $\partial_{X} H_{0}$ for the surface slope, we obtain

$$
\int_{-\infty}^{+\infty}\left[\partial_{X} H_{0}-\Theta(X) h_{0}^{\prime}\left(x_{\mathrm{TL}}\right)\right] d X=0 .
$$

Integrating this equation by parts we find

$$
\left[\tilde{H}_{0}\right]_{-\infty}^{+\infty}=0 .
$$

An intuitive graphical representation of this criterion is shown in Fig. 4(b). The triple-line position corresponds to the point where the linear extrapolations of the far-field profiles in the island and in the substrate regions meet. As a consequence of Eq. (69), we have $[h]_{\mathrm{TL}}=0$ to leading order and Eq. (65) reads

$$
v \mathcal{L}_{v}=[u]_{\mathrm{TL}} .
$$

This is the form of the kinetic boundary condition that will be used for further discussion and in the numerical analysis.

It is also useful to rewrite the kinetic coefficient $L_{v}$ via a change of variable to eliminate the variable $X$. Using Eq. (49), we rewrite Eq. (64) as

$$
\begin{aligned}
L_{v}= & \int_{0}^{H_{\mathrm{TL}}^{0}} \frac{2^{1 / 2}\left[W\left(H_{0}\right)-W(0)\right]^{1 / 2}}{\bar{\gamma}^{1 / 2} M\left(H_{0}\right)} d H_{0} \\
& +\int_{H_{\mathrm{TL}}^{0}}^{+\infty} \frac{d H_{0} 2^{1 / 2}}{\bar{\gamma}^{1 / 2}\left[W\left(H_{0}\right)-W(0)\right]^{1 / 2}} \\
& \times\left[\frac{W\left(H_{0}\right)-W(0)}{M\left(H_{0}\right)}-\frac{W(+\infty)-W(0)}{M(+\infty)}\right],
\end{aligned}
$$

where $H_{\mathrm{TL}}^{0}$ is the thickness of the film at the triple line to zeroth order. Using the Gibbs criterion (68), we obtain

$$
H_{\mathrm{TL}}^{0}+\int_{H_{\mathrm{TL}}^{0}}^{+\infty}\left[1-\left(\frac{W(\infty)-W(0)}{W\left(H_{0}\right)-W(0)}\right)^{1 / 2}\right] d H_{0}=0 .
$$

Using this equation, one can determine $H_{\mathrm{TL}}^{0}$ once the form of the wetting potential is known.

The first integral in Eq. (71) always converges when the substrate mobility is nonzero [42]. However, the finiteness of the second integral of Eq. (71) depends on the convergence of the wetting potential $W(H)$ and surface mobility $M(H)$ to their asymptotic values at large $H$. Consider that $W(H)$ and $M(H)$ converge to their corresponding far-field values as

$$
\begin{aligned}
& W(H)=W(\infty)+\delta W(H), \\
& M(H)=M(\infty)+\delta M(H),
\end{aligned}
$$

where $\delta W(H)$ and $\delta M(H) \rightarrow 0$ as $H \rightarrow+\infty$. Substituting these expressions in the second integral of Eq. (71), we find that $\mathcal{L}_{v}$ is finite only when $\delta W$ and $\delta M$ decrease faster than $1 / H$. Note also that Eq. (72) implies that the triple-line position is also only defined when $\delta W$ decreases faster than $1 / H$.

For most of the physical systems, the long-range behavior of the wetting potential is dominated by van der Waals interactions [41] with $W(H) \sim 1 / H^{2}$, leading to a finite value of $\mathcal{L}_{v}$. However, some debated works in metal films have suggested the possibility that $W \sim 1 / H$ due to electron confinement [22,43-45]. A contribution $W \sim 1 / H$ would also be present for an isolating film within a charged capacitor [46].

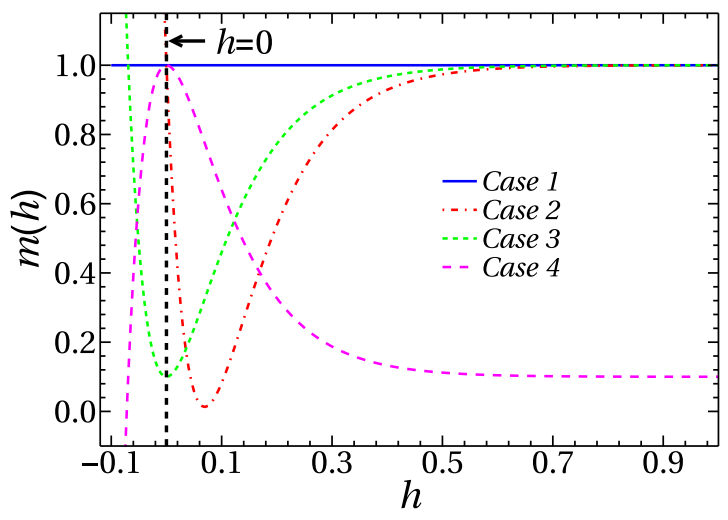

FIG. 3. Surface mobility as a function of film thickness for the four cases discussed in the text. The values of the parameters $p_{1}$ and $p_{2}$ for cases 3 and 4 are reported in Table I.

Concerning the mobility $M(H)$, we found no prediction of its possible dependence on the film thickness in the literature.

\section{Numerical simulations}

\section{Nonconserved dewetting}

In this section, we present numerical simulations of the nonconserved model (17). Our main purpose is to check the validity of the kinetic boundary condition (70). We use an exponentially decaying wetting potential

$$
w(h)=A_{1} e^{-h / h_{0}}-A_{2} e^{-h / 2 h_{0}},
$$

where $h_{0}$ determines the range of the potential. The parameters $A_{1}$ and $A_{2}$ can be determined from two conditions. First, the wetting potential is minimum at the substrate $w^{\prime}(0)=0$. Second, the equilibrium contact angle is imposed, leading to the condition $\gamma(+\infty) \cos \theta_{\mathrm{eq}}=\gamma(0)$. We then have

$$
A_{1}=\bar{\gamma}\left(1-\cos \theta_{\mathrm{eq}}\right), \quad A_{2}=2 A_{1} .
$$

We consider $\theta_{\mathrm{eq}}=10^{\circ}$, which ensures that the assumption of small slopes is satisfied, and $h_{0}=0.05$.

For the purpose of our numerical computations, it is convenient to define the mobility as a function of the wetting potential $w(h)$. We consider four different forms for the mobility $m(h)$. In the first case (case 1), the mobility is constant everywhere: $m(h)=1$. In the second case (case 2), the mobility is defined so as to decrease strongly in the triple-line region

$$
m(h)=1-\frac{w^{\prime}(h)}{\beta+w^{\prime}\left(h_{\mathrm{TL}}\right)},
$$

where $\beta$ is a small positive number. In the last two cases, we consider an asymmetric form of mobility, which has two different constant values in the far-field regions

$$
m(h)=p_{1}+p_{2} \frac{w(h)-w(0)}{w(\infty)-w(0)} .
$$

The parameters $p_{1}$ and $p_{2}$ can be chosen to obtain a higher mobility in the film region (case 3 ) or in the substrate region (case 4). The plots of mobility for these cases are shown in Fig. 3 with the parameters specified in Table I.

We chose to study the dewetting process of a thin film from a straight film edge. Assuming a constant contact angle, 
TABLE I. Kinetic coefficient $\mathcal{L}_{v}$ calculated using Eq. (71) for the four cases discussed in the text.

\begin{tabular}{lccc}
\hline \hline Case & Mobility & Parameters & $\mathcal{L}_{v}^{\text {th }}$ \\
\hline 1 & $M(h)=1.0$ & & -0.0174 \\
2 & Eq. (77) & $\beta=0.001$ & 0.218 \\
3 & Eq. (78) & $p_{1}=0.1, p_{2}=0.9$ & 0.0128 \\
4 & Eq. (78) & $p_{1}=1.0, p_{2}=-0.9$ & -0.535 \\
\hline \hline
\end{tabular}

Srolovitz and Safran [10] predicted a steady-state motion of the triple line with a constant velocity. We consider $\mu_{c}=0$ and a stepped initial profile defined as

$$
h(x, 0)=\bar{h}\left[\frac{1}{e^{\left(-x+x_{1}\right) / a}+1}+\frac{1}{e^{\left(x-x_{2}\right) / a}+1}-1\right],
$$

where $\bar{h}$ is the initial thickness of the film, $a$ is the initial width of the steps, and $x_{1}$ and $x_{2}$ are the positions of the steps with $x_{2}>x_{1}$. In our simulation, we have used $a=1$ and $\bar{h}=10 \gg$ $h_{0} \sim \epsilon$ to ensure a good separation of scales between the film region and the triple line.

\section{Numerical methods}

We use periodic boundary conditions and a pseudospectral method to solve Eq. (17) $[47,48]$. For this purpose, we rewrite Eq. (17) as

$$
\partial_{t} h=L[h]+N[h],
$$

where $L[h]$ and $N[h]$ represent the linear and nonlinear parts in $h$ :

$$
\begin{aligned}
L[h] & =\bar{\gamma} \partial_{x}^{2} h, \\
N[h] & =m(h)\left\{\frac{\mu_{c}}{\Omega}+\bar{\gamma} \partial_{x}^{2} h-w^{\prime}(h)\right\}-\bar{\gamma} \partial_{x}^{2} h .
\end{aligned}
$$

We take the Fourier transform of Eq. (80), which gives the following equation for a given mode $q$ in Fourier space:

$$
\partial_{t} \tilde{h}(q, t)=\tilde{L}(q) \tilde{h}(q, t)+\tilde{N}[\tilde{h}(q, t)] .
$$

In the simulations, we work in Fourier space and we update $\tilde{h}(q, t)$ at each time step. To calculate the Fourier transform of the first term in the nonlinear part $N[h]$, we first calculate the inverse Fourier transform of the term inside the curly brackets. Then we multiply it by $m(h)$ and take the Fourier transform of the resulting term.

We use two different time stepping methods. The first method is the implicit Euler method [49]. In this method, the linear part is calculated at the advanced time step $t+\Delta t$, whereas the nonlinear part is calculated at time $t$. This method corresponds to the following iterative scheme for Eq. (82):

$$
\tilde{h}(q, t+\Delta t)=\frac{\tilde{h}(q, t)+\Delta t \tilde{N}[\tilde{h}(q, t)]}{1+\tilde{L}(q) \Delta t} .
$$

Simulations are performed on a lattice of grid size $N=$ $2^{17}$, with grid spacing $\Delta x=\pi / 128$ and with the time step $\Delta t=0.01$. Fourier transforms are calculated via fast Fourier transform technique using the FFTw3 package [50].
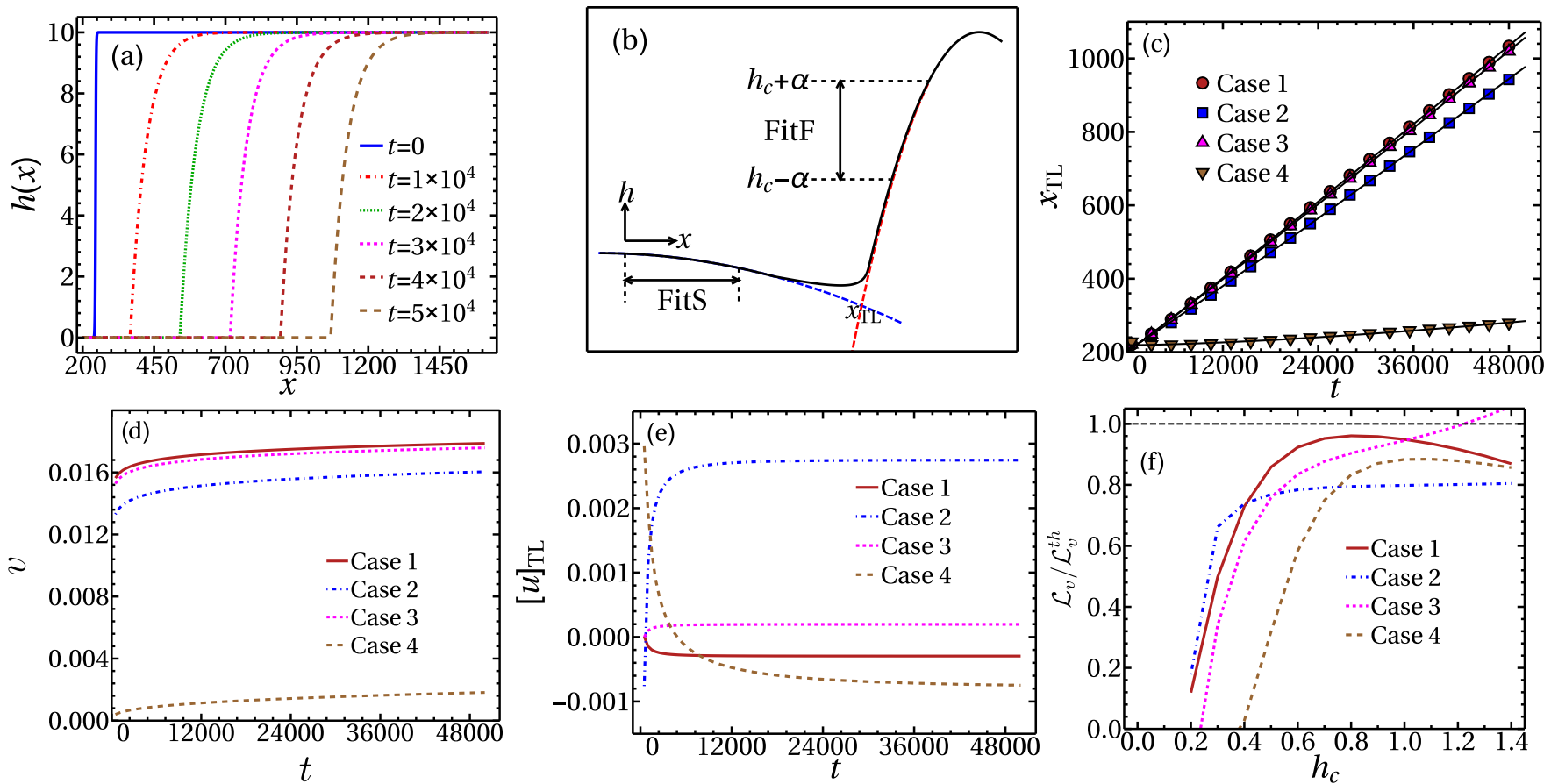

FIG. 4. Numerical simulation of the nonconserved model for $\mu_{c}=0$. (a) Time evolution of the film profile in the constant mobility case (case 1). (b) Schematic of the fitting procedure to find the triple-line position. The black solid curve represents the film profile. Blue and red curves are the functions in the island and substrate regions obtained by the fitting of the film profile in the fitting intervals FitS and FitF, respectively. Also shown is the time evolution of (c) the triple-line position $x_{\mathrm{TL}}$, (d) the triple-line velocity $v$, and (e) the thermodynamic force $[u]_{\mathrm{TL}}$. The four different cases correspond to four different forms of the mobility defined in the text. (f) Variation of the kinetic coefficients as a function of the center of the fitting region on the film side. The kinetic coefficients are normalized with respect to the theoretical prediction. 
The second method is an exponential time differencing method where time stepping is performed using a second-order Runge-Kutta method (allowing for large values of $\Delta t$ ). The iterative scheme for this method is discussed in Ref. [51]. The comparison of these two different methods allows us to check that the results are free from numerical artifacts.

\section{Results}

Figure 4(a) shows the evolution of film profile for a constant mobility. The film profile evolves through the evaporation of mass near the triple line. Due to the curvature of the film edge, the triple line recedes. Similar evolutions have been observed in the other three cases. However, the evolution becomes slower in the case where the mobility is small in the island region (case 4).

In order to calculate various quantities at the triple line, we determine the triple-line position from a fit of the film profile in the island and substrate regions using a quadratic function $a+$ $b x+c x^{2}$. The point of intersection of the extrapolation of these fits in the triple-line region gives the triple-line position [see Fig. 4(b)]. Figure 4(c) shows the time evolution of the tripleline position for the four above-mentioned cases of mobility. The plot of $x_{\mathrm{TL}}$ as a function of time is close to linear; in other words, the triple-line velocity is varying slowly with time as shown in Fig. 4(d).

To calculate the thermodynamic force $[u]_{\mathrm{TL}}$, we again fit the numerical data for $u$ in the island and substrate regions with the quadratic function $a+b x+c x^{2}$. The discontinuity of the extrapolated values of $u$ at the triple-line position provides $[u]_{\mathrm{TL}}$. The time evolution of $[u]_{\mathrm{TL}}$ is shown in Fig. 4(e). The kinetic coefficient $\mathcal{L}_{v}$ is then calculated using Eq. (70) from the ratio between $[u]_{\mathrm{TL}}$ and $v$.

Our results are sensitive to the choice of fitting interval in the island region. In order to make this dependence explicit, we consider fitting intervals of the form $h_{c}-0.1<h<h_{c}+0.1$, for different value of $h_{c}$, and calculate $\mathcal{L}_{v}$ in each case. Figure 4(f) shows the variation of $\mathcal{L}_{v} / \mathcal{L}_{v}^{\text {th }}$ with $h_{c}$, where $\mathcal{L}_{v}^{\text {th }}$ is calculated using the prediction of Eq. (71). For values of $h_{c}$ that are too close to the triple line, the fitting interval encroaches on the TL region, thereby making the fit less accurate. Indeed, we observe the decrease of $\mathcal{L}_{v}$ in all cases for small $h_{c}$. In contrast, as $h_{c}$ moves away from the triple line, the macroscopic curvature of the film leads to a deviation of the slope from its expected value close to the triple line. Globally, the kinetic coefficients obtained from simulations are in good agreement with the theoretical prediction (71) when $h_{c}$ is roughly 5-10 times the potential width $h_{0}$. The accuracy of $\mathcal{L}_{v}$ is then between $5 \%$ and $20 \%$.

\section{SURFACE DIFFUSION DYNAMICS}

\section{A. Model equations}

We now focus on the case of surface diffusion. Following the same strategy as in the nonconserved case, we use a generalization of the Mullins surface diffusion model [17] which accounts not only for a thickness-dependent interface energy [26,40,41], but also for a thickness-dependent mobility.

In this model, mass conservation impose that the normal velocity $v_{n}$ can be written as the divergence of a surface flux.
For the sake of simplicity, we write the model in one dimension

$$
v_{n}=-\partial_{s} j
$$

where $s$ is the arc length along the surface. The mass flux $j$ is proportional to gradients of chemical potential through the constitutive relation

$$
j=-b(h) \partial_{s} \frac{\mu}{\Omega},
$$

where $b(h)$ is the surface mobility. Substituting this expression in Eq. (84), we obtain

$$
\frac{1}{\left[1+\left(\partial_{x} h\right)^{2}\right]^{1 / 2}} \frac{\partial h}{\partial t}=\partial_{s}\left[b(h) \partial_{s} \frac{\mu}{\Omega}\right],
$$

where the local chemical potential is defined via Eq. (6). This model exhibits the same equilibrium properties as the nonconserved model characterized by a constant chemical potential $\mu$ and discussed in Sec. II. Also, following the same lines as in the nonconserved case, we use the small slope limit $\partial_{x} h \ll 1$, leading to

$$
\frac{\partial h}{\partial t}=\partial_{x}\left\{b(h) \partial_{x}\left[-\bar{\gamma} \partial_{x}^{2} h+\gamma^{\prime}(h)\right]\right\} .
$$

\section{B. Kinetic boundary condition}

\section{Matched asymptotic expansion}

Assuming again that the mobility and the potential vary on a scale $h \sim \epsilon$, we rewrite Eq. (87) using Eqs. (18) as

$$
\partial_{t} h=\partial_{x}\left[B(h / \epsilon) \partial_{x}\left(-\bar{\gamma} \partial_{x x} h+\frac{1}{\epsilon} W^{\prime}(h / \epsilon)\right)\right] .
$$

The procedure of the matched asymptotic expansion is then similar to that used in the nonconserved case. However, there is an important difference. Indeed, we now assume

$$
x=\chi / \epsilon, \quad h(x, t)=\epsilon^{2} \mathcal{H}(\chi, t)
$$

As compared to the choice used in the nonconserved case, such an expansion assumes smaller amplitudes and larger wavelengths for the deviations of the substrate profile from the minimum of the wetting potential. An inspection of the asymptotic expansion with the same scaling ansatz as in the nonconserved case (21) shows that the kinetic coefficients would then depend not only of $H_{0}$, but also on $H_{1}$. Since $H_{1}$ varies from one physical situation to the other, these kinetic coefficients would not be constant. Therefore, this option is not satisfactory. Instead, the scaling ansatz (89) provides kinetic coefficients that depend only on $H_{0}$, which is determined completely and solely by the functions $B(H)$ and $W(H)$. In addition, note that in the nonconserved case, the two choices of scaling ansatz in the substrate region lead to the same result. However, we prefer to use Eq. (21) because it provides a weaker condition for the variations of the surface profile in the substrate region.

The procedure of the asymptotic expansion is very similar to that of the nonconserved case. However, the calculations are lengthy and the details of the derivation are reported in Appendix A. Collecting all contributions up to third order, we finally obtain two linear kinetic boundary conditions, for the 
velocity and the flux at the triple line. These conditions can be written in two equivalent ways. The first form is

$$
\begin{gathered}
v \mathcal{L}_{2 v}+j_{-} \mathcal{L}_{1 v}=[u]_{\mathrm{TL}}-\mu_{+}[h]_{\mathrm{TL}}, \\
v \mathcal{L}_{1 v}+j_{-} \mathcal{L}_{1 J}=-[\mu]_{\mathrm{TL}}
\end{gathered}
$$

and the second form reads

$$
\begin{gathered}
-v\left(\overline{\mathcal{L}}_{2 v}-[h]_{\mathrm{TL}} \mathcal{L}_{2 J}\right)-j_{+} \mathcal{L}_{2 J}=[u]_{\mathrm{TL}}-\mu_{-}[h]_{\mathrm{TL}}, \\
-v \mathcal{L}_{2 J}+j_{+} \mathcal{L}_{1 J}=-[\mu]_{\mathrm{TL}},
\end{gathered}
$$

where $j_{-}=j\left(x \rightarrow x_{\mathrm{TL}}^{-}\right)$and $j_{+}=j\left(x \rightarrow x_{\mathrm{TL}}^{+}\right)$are, respectively, the mass fluxes at the triple line extrapolated from the far-field profile on the substrate and on the film side. In addition, the discontinuity of the mass flux at the triple line is related to the triple-line velocity $v$ via mass conservation:

$$
[j]_{\mathrm{TL}}=j_{+}-j_{-}=v[h]_{\mathrm{TL}} .
$$

The kinetic coefficients read

$$
\begin{aligned}
& \mathcal{L}_{1 J}=\epsilon L_{1 J}, \\
& \mathcal{L}_{1 v}=\epsilon^{2} L_{1 v}, \\
& \mathcal{L}_{2 v}=\epsilon^{3} L_{2 v},
\end{aligned}
$$

where

$$
\begin{gathered}
L_{1 J}=\int_{-\infty}^{+\infty} d X\left(\frac{1}{B\left(H_{0}\right)}-\frac{\Theta(X)}{B(\infty)}-\frac{\Theta(-X)}{B(0)}\right) \\
L_{1 v}=\int_{-\infty}^{+\infty} d X\left(\frac{H_{0}}{B\left(H_{0}\right)}-\Theta(X) \frac{X \partial_{x} h_{0}\left(x_{\mathrm{TL}}\right)+h_{1}\left(x_{\mathrm{TL}}\right)}{B(\infty)}\right) \\
L_{2 v}=\int_{-\infty}^{+\infty} d X\left[\frac{H_{0}^{2}}{B\left(H_{0}\right)}-\Theta(X) \frac{\left[X \partial_{x} h_{0}\left(x_{\mathrm{TL}}\right)+h_{1}\left(x_{\mathrm{TL}}\right)\right]^{2}}{B(\infty)}\right],
\end{gathered}
$$

and are related to the leading order by

$$
\begin{aligned}
& \mathcal{L}_{2 J}+\mathcal{L}_{1 v}=\mathcal{L}_{1 J}[h]_{\mathrm{TL}}, \\
& \overline{\mathcal{L}}_{2 v}+\mathcal{L}_{2 v}=\mathcal{L}_{1 v}[h]_{\mathrm{TL}} .
\end{aligned}
$$

The three relations (94), (101), and (102) allow one to pass from one of the forms of the kinetic boundary condition to the other.

Note that in both forms (90) and (91), or (92) and (93), the nondiagonal coefficients are equal. This symmetry property is a consequence of Onsager reciprocity relations. Due to these relations, we only have three independent kinetic coefficients.

\section{Definition of triple-line position}

Following the same procedure as in the nonconserved case, the triple-line position can be defined via a Gibbs dividing surfacelike relation $[h]_{\mathrm{TL}}=0$. Using Eqs. (101) and (102), we find that $\mathcal{L}_{2 J}=-\mathcal{L}_{1 v}$ and $\overline{\mathcal{L}}_{2 v}=-\mathcal{L}_{2 v}$. In addition, mass conservation (94) imposes the absence of discontinuity at the triple line $[j]_{\mathrm{TL}}=0$. The notation []$_{\mathrm{TL}}$ is the same as the notation []$_{-\infty}^{+\infty}$ defined in Appendix A. To leading order the two kinetic relations also simplify considerably and read

$$
\begin{gathered}
v \mathcal{L}_{2 v}+j \mathcal{L}_{1 v}=[u]_{\mathrm{TL}}, \\
v \mathcal{L}_{1 v}+j \mathcal{L}_{1 J}=-[\mu]_{\mathrm{TL}},
\end{gathered}
$$

where $j=j_{+}=j_{-}$. This compact form of the kinetic boundary conditions will be used in the following for the numerical analysis. Also notice that $h_{1}\left(x_{\mathrm{TL}}\right)=\left[\tilde{H}_{0}\right]_{-\infty}^{+\infty}=0$, leading to simpler expressions for $L_{1 v}$ and $L_{2 v}$.

\section{Kinetic coefficients}

We now rewrite Eqs. (98)-(100) without the variable $X$, making the dependence of the kinetic coefficients on the functions $W(H)$ and $B(H)$ explicit

$$
\begin{aligned}
L_{1 J} & =\int_{0}^{H_{\mathrm{TL}}^{0}} \frac{\bar{\gamma}^{1 / 2} d H_{0}}{2^{1 / 2}\left[W\left(H_{0}\right)-W(0)\right]^{1 / 2}}\left[\frac{1}{B\left(H_{0}\right)}-\frac{1}{B(0)}\right]+\int_{H_{\mathrm{TL}}^{0}}^{+\infty} \frac{\bar{\gamma}^{1 / 2} d H_{0}}{2^{1 / 2}\left[W\left(H_{0}\right)-W(0)\right]^{1 / 2}}\left[\frac{1}{B\left(H_{0}\right)}-\frac{1}{B(\infty)}\right], \\
L_{1 v} & =\int_{0}^{H_{\mathrm{TL}}^{0}} \frac{\bar{\gamma}^{1 / 2} d H_{0}}{2^{1 / 2}\left[W\left(H_{0}\right)-W(0)\right]^{1 / 2}} \frac{H_{0}}{B\left(H_{0}\right)}+\int_{H_{\mathrm{TL}}^{0}}^{+\infty} \frac{\bar{\gamma}^{1 / 2} d H_{0}}{2^{1 / 2}\left[W\left(H_{0}\right)-W(0)\right]^{1 / 2}}\left[\frac{H_{0}}{B\left(H_{0}\right)}-\frac{X\left(H_{0}\right) \partial_{x} h_{0}\left(x_{\mathrm{TL}}\right)}{B(\infty)}\right], \\
L_{2 v} & =\int_{0}^{H_{\mathrm{TL}}^{0}} \frac{\bar{\gamma}^{1 / 2} d H_{0}}{2^{1 / 2}\left[W\left(H_{0}\right)-W(0)\right]^{1 / 2}} \frac{H_{0}^{2}}{B\left(H_{0}\right)}+\int_{H_{\mathrm{TL}}^{0}}^{+\infty} \frac{\bar{\gamma}^{1 / 2} d H_{0}}{2^{1 / 2}\left[W\left(H_{0}\right)-W(0)\right]^{1 / 2}}\left[\frac{H_{0}^{2}}{B\left(H_{0}\right)}-\frac{\left[X\left(H_{0}\right) \partial_{x} h_{0}\left(x_{\mathrm{TL}}\right)\right]^{2}}{B(\infty)}\right],
\end{aligned}
$$

where the zeroth-order thickness of the film at the triple line $H_{\mathrm{TL}}^{0}$ is calculated from Eq. (72) and

$$
X\left(H_{0}\right) \partial_{x} h_{0}\left(x_{\mathrm{TL}}\right)=\int_{H_{\mathrm{TL}}^{0}}^{H_{0}} d H\left[\frac{W(\infty)-W(0)}{W(H)-W(0)}\right]^{1 / 2} .
$$

The first integral in the kinetic coefficients corresponds to the contribution of the substrate side. These integrals converge and are finite, except in the pathological case, where the mobility vanishes at the substrate.
The convergence of the second integral corresponding to the film side provides restrictive conditions on the thickness dependence of $W(H)$ and $B(H)$ for large $H$. We assume that $W(H)$ and $B(H)$ respectively tend to their corresponding far-field values in the film as $W(H)=W(\infty)+\delta W(H)$ and $B(H)=B(\infty)+\delta B(H)$, with $\delta W(H)$ and $\delta B(H) \rightarrow 0$ as $H \rightarrow+\infty$. Substituting these expressions in the above expressions of the kinetic coefficients, we find that the convergence of $\mathcal{L}_{1 J}$ requires that $\delta B(H)$ should decrease faster than $1 / H$. In addition, the convergence of $\mathcal{L}_{1 v}$ requires that $\delta W(H)$ 
TABLE II. Predicted values of kinetic coefficients from Eqs. (98)-(100).

\begin{tabular}{lllc}
\hline \hline Mobility & $\mathcal{L}_{2 v}$ & $\mathcal{L}_{1 v}$ & $\mathcal{L}_{1 J}$ \\
\hline case 1 & 0.014 & 0.094 & 0.0 \\
case 2 & 0.21 & 2.25 & 29.13 \\
case 3 & 0.037 & 0.29 & 0.0 \\
case 4 & 0.047 & 0.223 & 0.0 \\
\hline \hline
\end{tabular}

and $\delta B(H)$ should decrease faster than $1 / H^{2}$. Finally, the convergence of $\mathcal{L}_{2 v}$ requires that $\delta W(H)$ and $\delta B(H)$ should decrease faster than $1 / H^{3}$. Thus, overall, the existence of kinetic boundary conditions (103) and (104) require that $W(H)$ and $B(H)$ tend to their asymptotic values faster than $1 / H^{3}$. As a consequence, the generic decay of the wetting potential $W(H) \sim 1 / H^{2}$ coming from van der Waals interactions should lead to a divergence of the kinetic coefficients $\mathcal{L}_{1 v}$ and $\mathcal{L}_{2 v}$.

\section{Numerical simulations}

In order to check the predictions of the kinetic boundary conditions obtained from the asymptotic analysis, we perform numerical simulation of Eq. (86) using again the example of solid-state dewetting. The numerical scheme is the same as that discussed in Sec. III C2. However, in the conserved model, the dynamics is much slower. Therefore, a larger time step, which can be achieved by the second-order Runge-Kutta method discussed earlier, is necessary.
In the symmetric cases, we use the same expression of the wetting potential $w(h)$ and of the surface mobility $b(h)$ as in nonconserved dynamics. In the asymmetric cases, we choose a different form of the mobility which enforces a vanishing $L_{1 J}$. This choice allows us to calculate the smaller kinetic coefficients $\mathcal{L}_{2 v}$ and $\mathcal{L}_{1 v}$ more accurately. The predictions are reported in Table II. In case 3 , the mobility is defined by Eq. (78), with $p_{1} \approx 0.28$ and $p_{2} \approx 0.72$. In case 4 , the mobility is defined by

$$
b_{\text {as }}(h)=\frac{0.5}{0.5+\frac{1}{m(0)}-\frac{1}{m(h)}},
$$

where $m(h)$ is given by Eq. (78) with $p_{1} \approx 0.196$ and $p_{2} \approx 0.5$. Such an expression ensures that $L_{1 J}=0$.

Figure 5(a) shows the evolution of the film profile starting from an initial step profile [Eq. (79)]. As the triple line moves, there is an accumulation of mass near the triple line which leads to the formation of a rim. As we go away from the rim, the film profile exhibits spatial oscillations with decaying amplitude. The amplitude of oscillation increases with time, as discussed in details in Refs. [10,11].

Figure 5(b) describes the evolution of triple-line position with time. To calculate the triple-line position, we use the same procedure as that presented in the nonconserved case [see Fig. 4(b)]. We fit the triple-line position with the function

$$
x_{\mathrm{TL}}(t)=x_{0}+a t^{2 / 5}+b t^{1 / 5} .
$$

Such a time dependence for $x_{\mathrm{TL}}$ is derived from the Mullins model with a constant surface tension and a constant surface mobility, using boundary conditions with a fixed contact angle
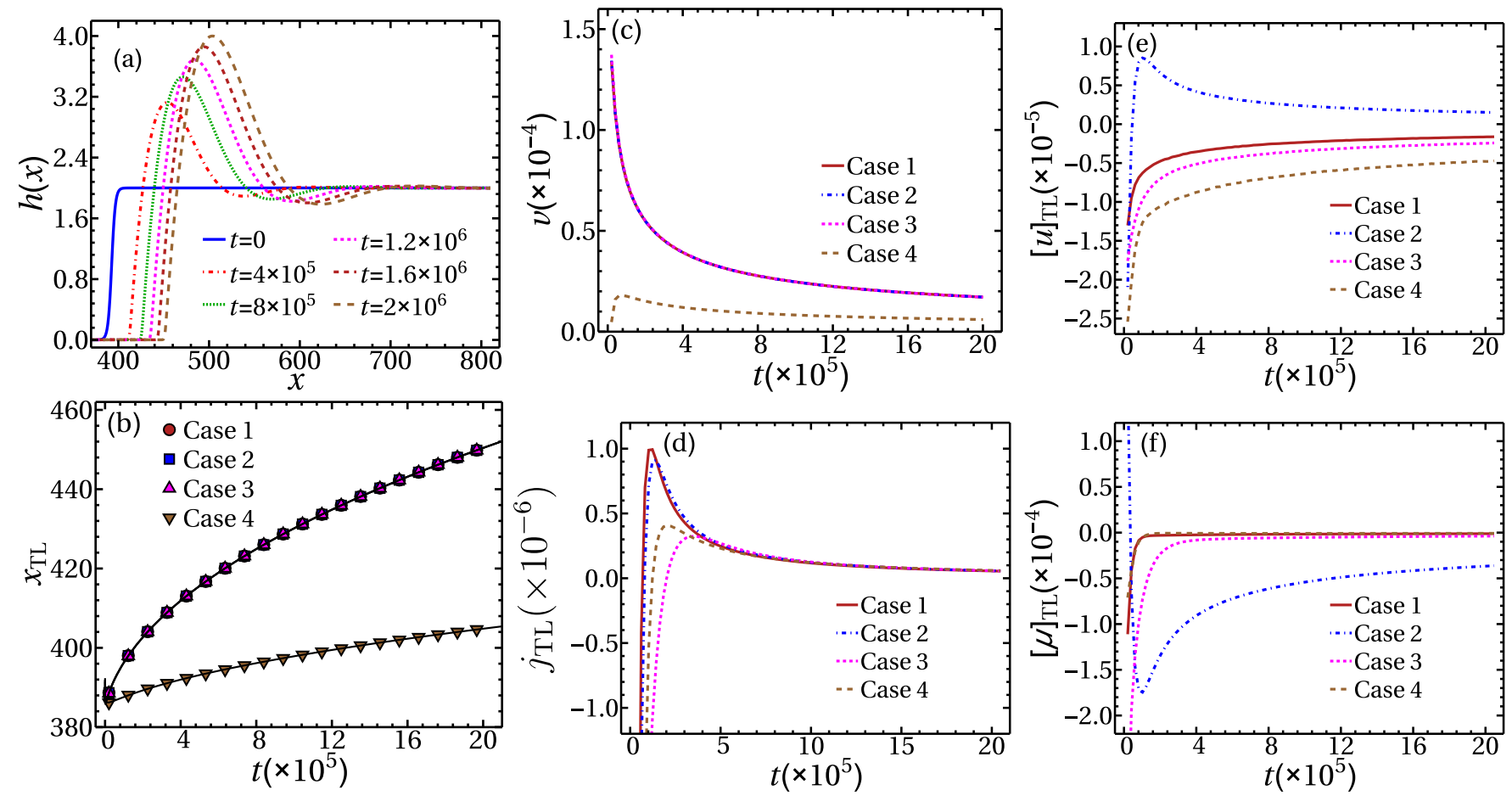

FIG. 5. Numerical results for the conserved model. (a) Evolution of the film profile starting from an step initial condition for constant mobility (case 1). (b) Time evolution of triple-line position for four different cases of surface mobility. Here symbols show the numerical data and corresponding black lines are the fit to the data according Eq. (110). Also shown is the temporal evolution of (c) the triple-line velocity $v$, (d) the mass flux $j_{\mathrm{TL}}$ approaching from the substrate side, (e) the thermodynamic force $[u]_{\mathrm{TL}}$, and (f) the difference in chemical potential $[\mu]_{\mathrm{TL}}$. 


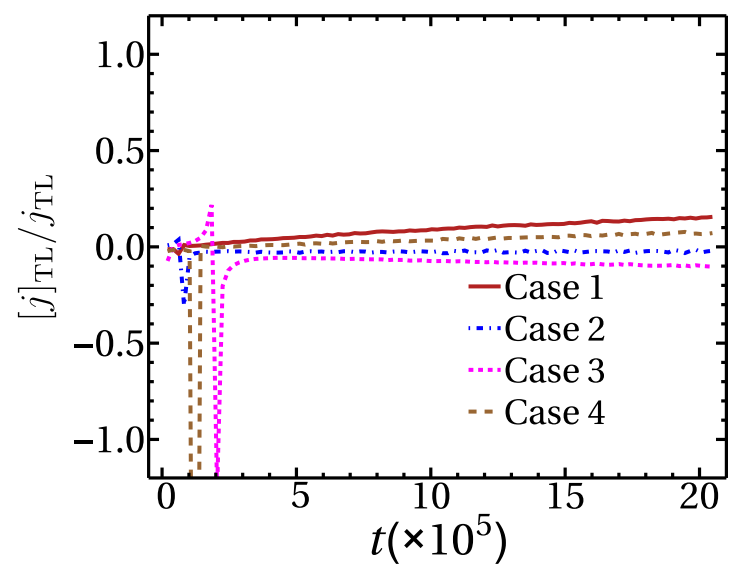

FIG. 6. Variation of $[j]_{\mathrm{TL}} / j_{\mathrm{TL}}$ with time. and vanishing mass flux [11]. The velocity of the triple line is then obtained by calculating the time derivative of $x_{\mathrm{TL}}$. This is shown in Fig. 5(c).

To calculate the quantities involved in kinetic boundary conditions (103) and (104), we again follow a procedure similar to that in Sec. III C 3. We first fit the relevant quantities in far-field regions with a quadratic function $a+b x+c x^{2}$. Then the discontinuity of these quantities is obtained by the extrapolation of these far-field fits to the triple line. As discussed in the case of the nonconserved model, the fitting procedure in the island region is very sensitive to the distance of the fitting region from the triple line. We therefore consider a range of fitting intervals $h_{c}-0.1<h<h_{c}+0.1$ for different value of $h_{c}$ and perform the minimization procedure for each $h_{c}$. Figures 5(d), 5(e), and 5(f) show the time evolution of $j_{\mathrm{TL}}$, $[u]_{\mathrm{TL}}$, and $[\mu]_{\mathrm{TL}}$, respectively, at the triple line with $h_{c}=0.5$.
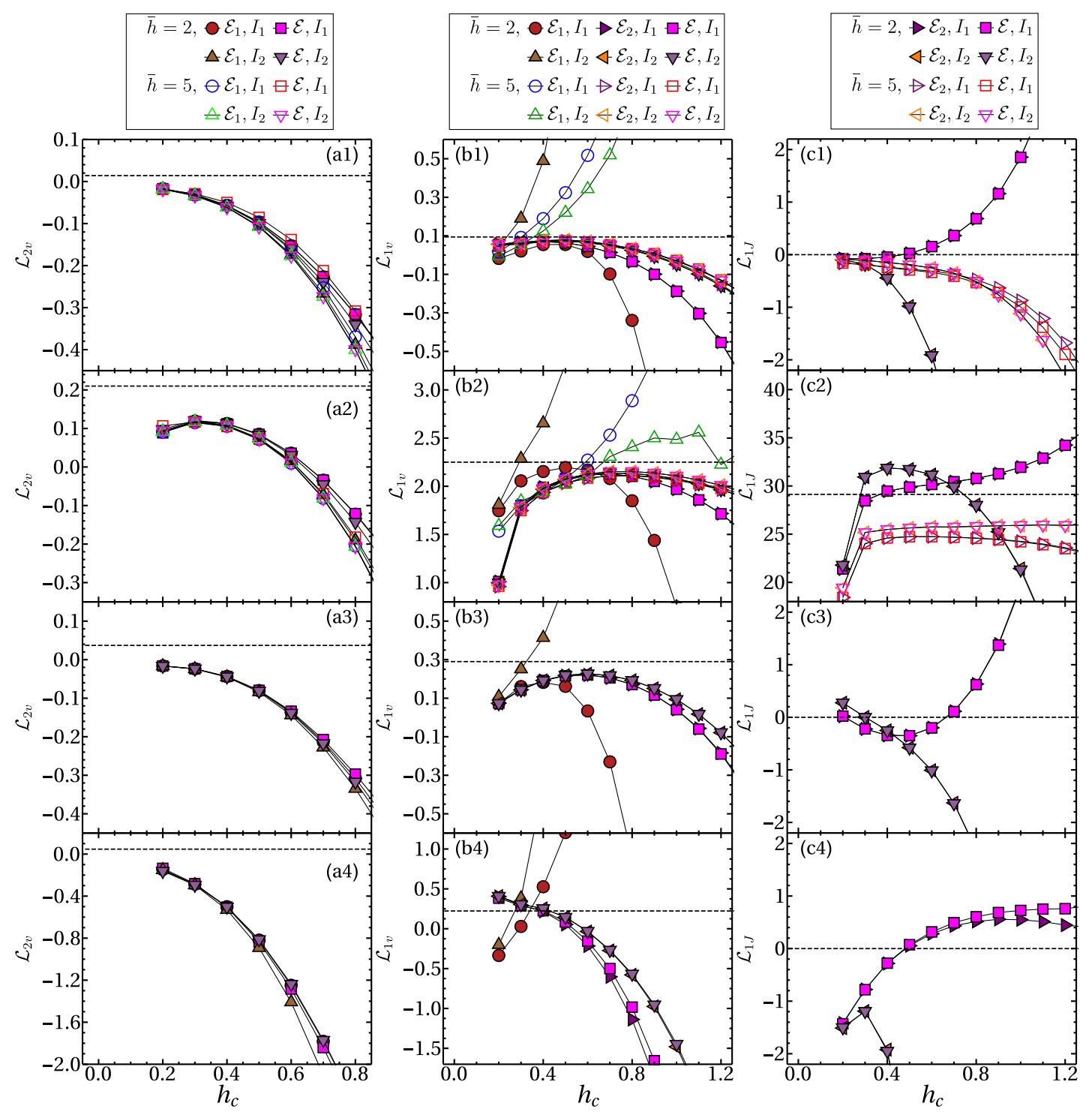

FIG. 7. Numerical verification of the kinetic boundary conditions. Here $j_{\mathrm{TL}}$ is the value obtained from the extrapolation of the mass flux from the substrate side. Closed and open symbols denote the data for the initial film thicknesses $\bar{h}=2$ and $\bar{h}=5$, respectively. In addition, $\mathcal{E}_{1}, \mathcal{E}_{2}$, and $\mathcal{E}$ are the time-averaged errors defined in the text. Averages are performed in the intervals $I_{1}$ with $2 \times 10^{4}<t<2 \times 10^{6}$ and $I_{2}$ with $2 \times 10^{5}<t<2 \times 10^{6}$. The legend for each column is shown on top. The dashed black line in each plot corresponds to the analytical prediction reported in Table II. As discussed in the text, the four panels in each column correspond to the four different mobility cases. 


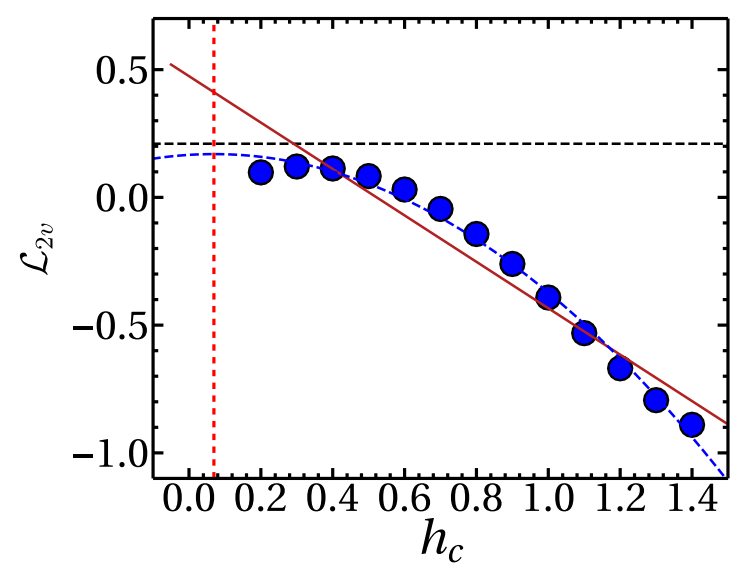

FIG. 8. Convergence of the numerical procedure to extract $\mathcal{L}_{2 v}$ for case 2. Fit of numerical data for $\mathcal{L}_{2 v}$, using linear (solid red line) and quadratic (dashed blue curve) functions described in the text. The horizontal dashed line shows the predicted value. The vertical dashed line shows the position of the triple line.

As evident from Fig. 5, there is an initial transient regime with large variations in $[u]_{\mathrm{TL}},[\mu]_{\mathrm{TL}}$, and $j_{\mathrm{TL}}$. Also, the difference $[j]_{\mathrm{TL}} / j_{\mathrm{TL}}$ is large at short times, violating the local mass conservation relation (94), and becomes small only at long times, as shown in Fig. 6. This observation suggests that at short times there is a contribution from the time dependence of the profile which could be too fast to be caught by our asymptotic analysis.

Bearing this in mind, we have analyzed the kinetic boundary conditions in two time intervals: The first interval is $I_{1}=2 \times$ $10^{4}<t<2 \times 10^{6}$, which includes the short-time regime, and the second interval is $I_{2}=2 \times 10^{5}<t<2 \times 10^{6}$, where we have slow motion of the triple line. Within each time interval, we evaluate the kinetic coefficients from the kinetic boundary conditions (103) and (104). Since the convergence of a fit with three parameters $\left(\mathcal{L}_{1 v}, \mathcal{L}_{2 v}\right.$, and $\left.\mathcal{L}_{1 J}\right)$ is more delicate than in the nonconserved case, we now use a systematic approach based on the error estimates

$$
\begin{aligned}
\mathcal{E}_{1}\left(\mathcal{L}_{2 v}, \mathcal{L}_{1 v}\right) & =\int\left(v \mathcal{L}_{2 v}+j \mathcal{L}_{1 v}-[u]_{\mathrm{TL}}\right)^{2} d t, \\
\mathcal{E}_{2}\left(\mathcal{L}_{1 v}, \mathcal{L}_{1 J}\right) & =\int\left(v \mathcal{L}_{1 v}+j \mathcal{L}_{1 J}+[\mu]_{\mathrm{TL}}\right)^{2} d t, \\
\mathcal{E}\left(\mathcal{L}_{2 v}, \mathcal{L}_{1 v}, \mathcal{L}_{1 J}\right) & =\mathcal{E}_{1}\left(\mathcal{L}_{2 v}, \mathcal{L}_{1 v}\right)+\mathcal{E}_{2}\left(\mathcal{L}_{1 v}, \mathcal{L}_{1 J}\right) .
\end{aligned}
$$

The first two equations define the cumulative deviations from the kinetic boundary conditions (103) and (104). We find numerically the values of the kinetic coefficients which minimize these errors.

The nonzero value of $[j]_{\mathrm{TL}}$ also leads to an ambiguity in the choice of $j$ in the kinetic relations, where the mass flux is the same on both sides of the triple line. In order to probe the consequences of this ambiguity, we consider both values of $j$, viz., the values obtained by extrapolating the mass flux at the triple line from the substrate and island regions. Figure 7 represents the kinetic coefficients calculated from the value of $j$ on the substrate side. These figures show the variation of kinetic coefficients with $h_{c}$ for the four different mobilities. For the first two cases, viz., for cases 1 and 2 , we report the result for two initial thicknesses of the film, $\bar{h}=2$ and $\bar{h}=5$. In cases 3 and 4, results are reported for $\bar{h}=2$.

Results for the two different film thicknesses indicate that the kinetic coefficients are not sensitive to the film thickness as long as $\bar{h} \gg h_{0}$ (i.e., $\epsilon \ll 1$ ). Similar results are obtained when using the value of $j$ from the film side (results are presented in Appendix B, in Fig. 11).

Globally, we found fair agreement between the theoretical and numerical results, especially at small and intermediate values of $h_{c}$ for $\mathcal{L}_{1 J}$ and $\mathcal{L}_{1 v}$. However, although it exhibits the expected trends, the convergence of $\mathcal{L}_{2 v}$ is less quantitative.

The first column of Fig. 7 shows the variation of $\mathcal{L}_{2 v}$ with $h_{c}$. It is clear from the figures that $\mathcal{L}_{2 v}$ converges only for small values of $h_{c}$. Deviations are observed when we shift the fitting region away from the triple line. However, as we approach the triple line, the wetting potential starts to affect various quantities, as can be seen clearly, e.g., in Fig. 7(a2). In Fig. 8, we have fitted $\mathcal{L}_{2 v}$ as a function of $h_{c}$ with linear and quadratic fits of the forms $a+b\left(h_{c}-h_{\mathrm{TL}}\right)$ and $a+b\left(h_{c}-h_{\mathrm{TL}}\right)^{2}$, where $h_{\mathrm{TL}}$ is the triple-line position to leading order. An extrapolation of these fits towards the triple line indicates that $\mathcal{L}_{2 v}$ shows a convergence consistent with the theoretical value. A similar trend can also be seen for the kinetic coefficients $\mathcal{L}_{1 v}$ and $\mathcal{L}_{1 J}$. However, in these cases, a simple extraction of the values obtained in the range $0.4<h_{c}<0.5$ provides reasonable agreement with the asymptotic analysis in all cases.

Note also that the value of the kinetic cross term $\mathcal{L}_{1 v}$ obtained by minimization of $\mathcal{E}_{2}$ shows a relatively better convergence as compared to the value obtained with $\mathcal{E}_{1}$. This is probably caused by the coupling to $\mathcal{L}_{2 v}$ in $\mathcal{E}_{1}$, which exhibits a poorer convergence.
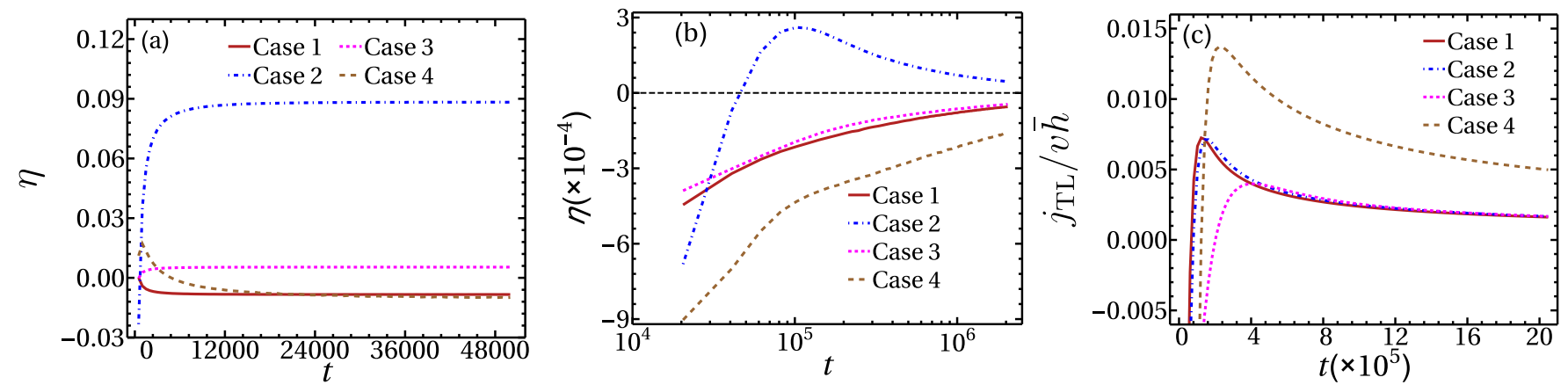

FIG. 9. Relative deviation of the contact angle and mass flux balance during dewetting. The relative deviation of the dynamic contact angle from its equilibrium value is shown for (a) the nonconserved and (b) the conserved models. (c) Comparison of the mass flux through the triple line to the mass flux corresponding to the increase of the volume of the rim resulting from its motion at velocity $v$. 


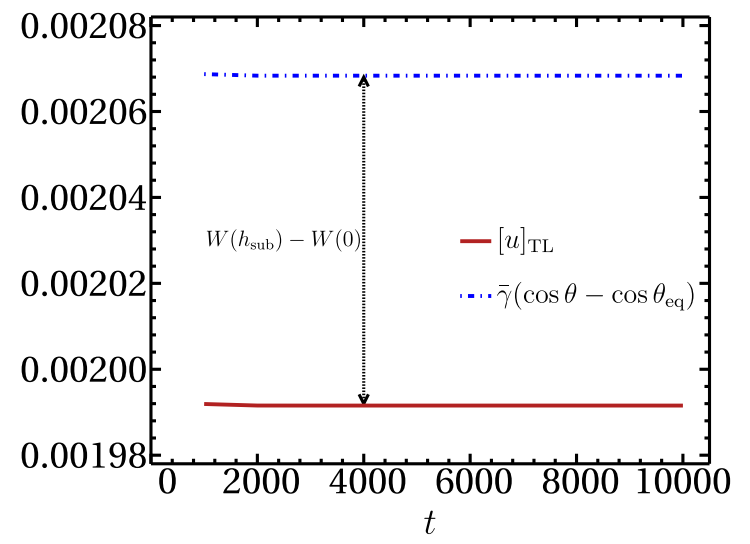

FIG. 10. Decomposition of $[u]_{\mathrm{TL}}$ following Eq. (113) for the nonconserved model. The simulation was performed with a vapor chemical potential $\mu_{c}=0.02$.

\section{DISCUSSION}

We define the relative deviation of dynamic contact angle $\theta_{D}$ from its equilibrium value

$$
\eta=\left(\theta_{\mathrm{eq}}-\theta_{D}\right) / \theta_{\mathrm{eq}} \text {. }
$$

This quantity can be measured during dewetting for nonconserved and conserved models, as shown in Figs. 9(a) and 9(b), respectively. In order to obtain $\eta$ from simulations, we have extracted $\theta_{D}$ from $[u]_{\mathrm{TL}}$ using Eq. (46). In the nonconserved case, $\eta$ could reach up to $10 \%$. However, as expected in conserved dewetting dynamics where the triple line slows down with time after the initial transient phase, $\theta_{D}$ converges towards the equilibrium contact angle. The deviation in our simulations is small. We found $\eta \sim 10^{-3}-10^{-4}$ in conserved dynamics. This quantity has been measured in experiments in liquid polymer films [4,5], but it has not been measured yet in the case of solids.

We have also measured the ratio $j_{\mathrm{TL}} / v h$, which quantifies the relevance of the mass flux at the triple line $j_{\mathrm{TL}}$ as compared to the typical rate $v h$ at which mass from the film is incorporated in the dewetting rim. The result is shown in Fig. 9(c). This ratio decreases with time. However, it exhibits an early-time maximum around 1\%. As discussed in Ref. [52], the mass flux at the triple-line could be much more important in situations where evaporation occurs mainly in the substrate region. Indeed, in this case, mass diffuses from the film to the substrate before evaporating from the substrate, leading to a significant mass flux through the triple line.

As a final remark, one should note the difference between $[u]_{\mathrm{TL}}$ and the deviation of the contact angle from the Young equilibrium angle. Assuming a triple-line definition with $[h]_{\mathrm{TL}}=0$ and combining the equilibrium equations (13) and (14) and the definition of $u$ [Eq. (46)] in the small slope limit where $\partial_{x} h \sim \theta$, one finds two contributions to the discontinuity of $u$ at the triple line

$$
\begin{aligned}
{[u]_{\mathrm{TL}} } & \approx \bar{\gamma}\left(\cos \theta_{D}-\cos \theta_{\mathrm{eq}}\right)-w\left(h_{\mathrm{sub}}\right)+w(0) \\
& \approx \bar{\gamma}\left(\cos \theta_{D}-\cos \theta_{\mathrm{TL}}^{\mathrm{eq}}\right) .
\end{aligned}
$$

To obtain these contributions, we have also assumed that the angle on the substrate side is vanishingly small. As a consequence, $[u]_{\mathrm{TL}}$ does not describe the deviation from the equilibrium Young contact angle $\theta_{\mathrm{eq}}$, but the deviation from the contact angle $\theta_{\mathrm{TL}}^{\mathrm{eq}}$ which accounts for the fact that the substrate height might not be the equilibrium height, thereby leading to a shift in the substrate free energy. This difference can be significant, for example, in growth when $\mu_{c} \neq 0$. To verify this, we have simulated the nonconserved model with a nonzero chemical potential in the vapor phase $\mu_{c}=0.02$. Figure 10 confirms that $[u]_{\mathrm{TL}}$ exhibits both contributions, viz., the deviation of the contact angle from the Young equilibrium value and the departure from equilibrium height in the substrate region.

\section{CONCLUSION}

As a summary, we have derived nonequilibrium kinetic boundary conditions at the triple line for solid-state wetting, considering both nonconserved (evaporation-condensation) and conserved (surface diffusion) dynamics. This result was obtain by means of a matched asymptotic expansion from an isotropic mesoscopic continuum model with a wetting potential and a thickness-dependent mobility.

In the nonconserved model, we obtained a single kinetic boundary condition, which relates the triple-line velocity to the deviation of the contact angle from its equilibrium value. For the conserved model we found that not only does the contact angle deviate from its equilibrium value, but there is also a nonzero surface diffusion mass flux at the triple line. This gives rise to two kinetic boundary conditions, which relate the two fluxes, i.e., velocity and mass fluxes, to two driving forces, i.e., contact angle deviation and discontinuity of the chemical potential (with cross terms).

We have verified the validity of these kinetic boundary conditions through numerical simulation of solid-state dewetting. These simulations show reasonable agreement with kinetic boundary conditions for both cases. However, quantitative agreement is more difficult to reach in the conserved case.

The mesoscopic continuum model presented here is an efficient tool to perform numerical simulations of solid-state dewetting or of other processes involving the evolution of thinfilm morphologies such as growth, sublimation, spreading, Ostwald ripening [26], or the Rayleigh-Plateau instability [53]. It has the advantage of including intrinsically the boundary conditions at the triple line, leading to an interface capturing method which avoids the delicate procedures required to track the triple line and to extrapolate the interface profile at the triple line in order to impose the boundary conditions. In addition, this two-dimensional model exhibits a reduced dimensionality, as compared to three-dimensional phase field models, which should lead to significant simulation-time reduction. Furthermore, anisotropy can easily be included in mesoscopic continuum models following a procedure similar to that in phase field models [54]. To some extent, the anisotropy of the surface free energy and of the mobility can be derived from step models [55].

Finally, the kinetic coefficients are finite only for wetting potentials and mobilities that converge fast enough to their asymptotic values for thick films. When these converge too slowly, the kinetic coefficients diverge. Such a divergence is found, for example, for conserved dynamics with a van der Waals wetting potential decaying as $1 / h^{2}$. This divergence of diffusive dissipation seems a priori to share similarities with 
the divergence of viscous dissipation at the triple line of thin liquid films [3]. However, the situation is different because the divergence occurs at small scales for liquid films, while it occurs at large scales for solid films. Note that divergences induced by long-range potentials have also been observed in static thermodynamic liquid interface properties [56].

At this stage of our understanding, the question is the following: In the cases where the kinetic coefficients are not defined (diverge), is it possible to change the technical procedure of the expansion to obtain suitable effective boundary conditions, or is this divergence a physical effect, indicating that the boundary conditions involving contact angles and nonequilibrium mass fluxes cannot be defined? Further investigations are needed to clarify this issue.

\section{ACKNOWLEDGMENT}

We acknowledge support from the ANR LOTUS Grant No. ANR-13-BS04-0004.

\section{APPENDIX A: DERIVATION OF THE KINETIC BOUNDARY CONDITIONS FOR THE CONSERVED MODEL}

Using the scaling defined by Eq. (89) in the substrate region, the matching condition between triple line and substrate reads

$$
\epsilon H(x, t ; \epsilon)=\epsilon^{2} \mathcal{H}(\chi, t ; \epsilon) .
$$

Expanding the matching condition using Eqs. (29) and (30), we obtain

$$
\begin{gathered}
H_{0}(X, t)=0 \\
H_{1}(X, t)=\mathcal{H}_{0}\left(\chi_{\mathrm{TL}}\right), \\
H_{2}(X, t)=\mathcal{H}_{1}\left(\chi_{\mathrm{TL}}\right), \\
H_{3}(X, t)=X \partial_{\chi} \mathcal{H}_{0}\left(\chi_{\mathrm{TL}}\right)+\mathcal{H}_{2}\left(\chi_{\mathrm{TL}}\right), \\
H_{4}(X, t)=X \partial_{\chi} \mathcal{H}_{1}\left(\chi_{\mathrm{TL}}\right)+\mathcal{H}_{3}\left(\chi_{\mathrm{TL}}\right) .
\end{gathered}
$$

Following the same procedure as for the nonconserved model, we write Eq. (88) in the island, triple-line, and substrate regions as, respectively,

$$
\begin{gathered}
\partial_{t} h-v \partial_{x} h=-\bar{\gamma} B(\infty) \partial_{x}^{4} h(x, t), \\
\epsilon^{4} \partial_{t} H-\epsilon^{3} v \partial_{X} H=\partial_{X}\left\{B(H) \partial_{X}\left[-\bar{\gamma} \partial_{X}^{2} H+W^{\prime}(H)\right]\right\}, \\
\partial_{t} \mathcal{H}-v \partial_{\chi} \mathcal{H}=\partial_{\chi}\left[B(\epsilon \mathcal{H}) \partial_{\chi}\left(-\bar{\gamma} \epsilon^{4} \partial_{\chi}^{2} \mathcal{H}+\frac{1}{\epsilon} W^{\prime}(\epsilon \mathcal{H})\right)\right] .
\end{gathered}
$$

\section{The TL region}

We now study the film profile in the TL region. For this purpose, we integrate Eq. (A8). This gives

$\epsilon^{4} \int_{X_{-}}^{X} d X^{\prime} \partial_{t} H^{\prime}-\epsilon^{3} v\left[H(X)-H\left(X_{-}\right)\right]=-J(X)+J\left(X_{-}\right)$, where $J(X)=B(H) \partial_{X}\left[\bar{\gamma} \partial_{X}^{2} H-W^{\prime}(H)\right]$ is the surface diffusion mass flux. A second integration leads to

$$
\begin{gathered}
\epsilon^{4} \int_{X_{-}}^{X} \frac{d X^{\prime}}{B\left(H^{\prime}\right)} \int_{X_{-}}^{X^{\prime}} d X^{\prime \prime} \partial_{t} H^{\prime \prime}-\epsilon^{3} v \int_{X_{-}}^{X} d X^{\prime} \frac{H^{\prime}-H\left(X_{-}\right)}{B\left(H^{\prime}\right)} \\
=v(X)-v\left(X_{-}\right)+J\left(X_{-}\right) \int_{X_{-}}^{X} \frac{d X^{\prime}}{B\left(H^{\prime}\right)},
\end{gathered}
$$

where $H^{\prime}=H\left(X^{\prime}\right)$ and

$$
v(X)=-\bar{\gamma} \partial_{X}^{2} H+W^{\prime}(H)
$$

is the chemical potential in triple-line variables. Multiplying Eq. (A11) by $\partial_{X} H$ and integrating, we find a third relation

$$
\begin{gathered}
\epsilon^{4} \int_{X_{-}}^{X} d X^{\prime} \partial_{X^{\prime}} H^{\prime} \int_{X_{-}}^{X^{\prime}} \frac{d X^{\prime \prime}}{B\left(H^{\prime \prime}\right)} \int_{X_{-}}^{X^{\prime \prime}} d X^{\prime \prime \prime} \partial_{t} H^{\prime \prime \prime} \\
-\epsilon^{3} v \int_{X_{-}}^{X} d X^{\prime} \partial_{X^{\prime}} H^{\prime} \int_{X_{-}}^{X^{\prime}} d X^{\prime \prime} \frac{H^{\prime \prime}-H\left(X_{-}\right)}{B\left(H^{\prime \prime}\right)} \\
=U(X)-U\left(X_{-}\right)-v\left(X_{-}\right) \int_{X_{-}}^{X} d X^{\prime} \partial_{X^{\prime}} H^{\prime} \\
\quad+J\left(X_{-}\right) \int_{X_{-}}^{X} d X^{\prime} \partial_{X^{\prime}} H^{\prime} \int_{X_{-}}^{X^{\prime}} \frac{d X^{\prime \prime}}{B\left(H^{\prime \prime}\right)} .
\end{gathered}
$$

Below, we expand Eqs. (A8) and (A13) and solve the resulting equations to each order in $\epsilon$.

\section{a. Zeroth order}

To leading order Eq. (A8) reads

$$
\partial_{X}\left\{B\left(H_{0}\right) \partial_{X}\left[-\bar{\gamma} \partial_{X}^{2} H_{0}+W^{\prime}\left(H_{0}\right)\right]\right\}=0 .
$$

Integrating this equation, we find

$$
B\left(H_{0}\right) \partial_{X}\left[-\bar{\gamma} \partial_{X}^{2} H_{0}+W^{\prime}\left(H_{0}\right)\right]=J_{0} .
$$

From the derivatives of the matching condition (33) with respect to $X$ as $X \rightarrow+\infty$, we obtain

$$
\begin{aligned}
\partial_{X}^{3} H_{0} & \rightarrow 0, \\
\partial_{X}\left[W^{\prime}\left(H_{0}\right)\right]=W^{\prime \prime}\left(H_{0}\right) \partial_{X} H_{0} & \rightarrow 0,
\end{aligned}
$$

which implies $J_{0}=0$. Here we have used the fact that $W^{\prime \prime}$ vanishes as $X \rightarrow+\infty$.

Similarly, as $X \rightarrow-\infty$, using the matching condition(A2), we find

$$
\begin{aligned}
\partial_{X}^{3} H_{0} & \rightarrow 0, \\
\partial_{X}\left[W^{\prime}\left(H_{0}\right)\right]=W^{\prime \prime}(0) \partial_{X} H_{0} & \rightarrow 0,
\end{aligned}
$$

which again implies $J_{0}=0$. Thus we have

$$
\partial_{X}\left[-\bar{\gamma} \partial_{X}^{2} H_{0}+W^{\prime}\left(H_{0}\right)\right]=0 .
$$

Integrating this equation once again, we find

$$
-\bar{\gamma} \partial_{X}^{2} H_{0}+W^{\prime}\left(H_{0}\right)=v_{0} .
$$

Using the matching condition, as $X \rightarrow+\infty$, we obtain

$$
\left.\begin{array}{rl}
\partial_{X}^{2} H_{0} & \rightarrow 0 \\
W^{\prime}\left(H_{0}\right)=W^{\prime}(\infty) & \rightarrow 0
\end{array}\right\} \Rightarrow v_{0}=0 .
$$


Also, as $X \rightarrow-\infty$, we have

$$
\left.\begin{array}{r}
\partial_{X}^{2} H_{0} \rightarrow 0 \\
W^{\prime}\left(H_{0}\right)=W^{\prime}(0)=0
\end{array}\right\} \Rightarrow v_{0}=0 .
$$

Thus Eq. (A19) reads

$$
-\bar{\gamma} \partial_{X}^{2} H_{0}+W^{\prime}\left(H_{0}\right)=0 .
$$

We now consider Eq. (A13) to zeroth order, which may be rewritten as

$$
\left[U_{0}\right]_{-\infty}^{+\infty}-v_{0}(-\infty)\left[H_{0}\right]_{-\infty}^{+\infty}=0
$$

with $U_{0}$ given by

$$
U_{0}(X)=-\frac{\bar{\gamma}}{2}\left(\partial_{X} H_{0}\right)^{2}+W\left(H_{0}\right)
$$

Since $v_{0}(-\infty)=0$ from Eq. (A21), we obtain

$$
\left[U_{0}\right]_{-\infty}^{+\infty}=0
$$

Following the same lines as in the nonconserved case, we then obtain Young's contact angle relation (1) using matching conditions.

\section{b. First order}

To first order, Eq. (A8) leads to

$$
\begin{aligned}
& \partial_{X}\left\{B\left(H_{0}\right) \partial_{X}\left[-\bar{\gamma} \partial_{X}^{2} H_{1}+W^{\prime \prime}\left(H_{0}\right) H_{1}\right]\right\} \\
& \quad+\partial_{X}\left\{B^{\prime}\left(H_{0}\right) H_{1} \partial_{X}\left[-\bar{\gamma} \partial_{X}^{2} H_{0}+W^{\prime}\left(H_{0}\right)\right]\right\}=0 .
\end{aligned}
$$

Since the mass flux to zeroth order is zero, the last term in Eq. (A26) vanishes. An integration of the resulting equation, combined with the matching conditions in the far-field limits $\left(X \rightarrow \pm \infty\right.$ ), shows that the mass flux to first order $J_{1}=0$ vanishes. Further integration of the resulting equation leads to an expression of the chemical potential to first order

$$
-\bar{\gamma} \partial_{X}^{2} H_{1}+W^{\prime \prime}\left(H_{0}\right) H_{1}=v_{1} .
$$

Again using matching conditions, we obtain

$$
v_{1}=-\bar{\gamma} \partial_{x}^{2} h_{0}\left(x_{\mathrm{TL}}\right)=W^{\prime \prime}(0) \mathcal{H}_{0}\left(\chi_{\mathrm{TL}}\right) .
$$

This relation implies that the deviation $\mathcal{H}_{0}\left(\chi_{\mathrm{TL}}\right)$ of $h$ around the minimum of the wetting potential at the substrate is related to the film curvature $\partial_{x}^{2} h_{0}\left(x_{\mathrm{TL}}\right)$ at the triple line. This relation was obtained in Sec. II at equilibrium. Hence, to this order we obtain the equilibrium relations with zero mass flux and constant chemical potential.

Moreover, Eq. (A13) to first order reads

$$
\left[U_{1}\right]_{-\infty}^{+\infty}-\tilde{v}_{0}(-\infty)\left[H_{1}\right]_{-\infty}^{+\infty}-\tilde{v}_{1}(-\infty)\left[H_{0}\right]_{-\infty}^{+\infty}=0
$$

with $U_{1}(X)$ defined as

$$
U_{1}(X)=-\bar{\gamma}\left(\partial_{X} H_{0}\right)\left(\partial_{X} H_{1}\right)+W^{\prime}\left(H_{0}\right) H_{1} .
$$

Using Eqs. (A21) and (A28) and matching conditions, we find that diverging terms cancel and we obtain the first-order correction to the equilibrium Young contact angle relation

$$
\left[\tilde{U}_{1}\right]_{-\infty}^{+\infty}-\tilde{v}_{0}(-\infty)\left[\tilde{H}_{1}\right]_{-\infty}^{+\infty}-\tilde{v}_{1}(-\infty)\left[\tilde{H}_{0}\right]_{-\infty}^{+\infty}=0
$$

\section{c. Second order}

To second order, from Eq. (A8) we obtain

$$
\begin{aligned}
& \partial_{X}\left[B ( H _ { 0 } ) \partial _ { X } \left(-\bar{\gamma} \partial_{X}^{2} H_{2}+W^{\prime \prime}\left(H_{0}\right) H_{2}\right.\right. \\
& \left.\left.+W^{\prime \prime \prime}\left(H_{0}\right) \frac{H_{1}^{2}}{2}\right)\right]=0 .
\end{aligned}
$$

Here we have used the fact that the mass flux vanishes to zeroth and first order, so the two other terms appearing in Eq. (A32) vanish. Integrating this equation, we obtain

$$
B\left(H_{0}\right) \partial_{X}\left(\bar{\gamma} \partial_{X}^{2} H_{2}-W^{\prime \prime}\left(H_{0}\right) H_{2}-W^{\prime \prime \prime}\left(H_{0}\right) \frac{H_{1}^{2}}{2}\right)=J_{2}
$$

As $X \rightarrow-\infty$, using Eqs. (A2)-(A4), we have

$$
J_{2}=0 \text {. }
$$

Similarly matching the condition at $X \rightarrow+\infty$ gives

$$
J_{2}=\bar{\gamma} B(\infty) \partial_{x}^{3} h_{0}\left(x_{\mathrm{TL}}\right)=0 .
$$

Therefore, we have no flow of mass to this order. Integrating Eq. (A33), we obtain

$$
-\bar{\gamma} \partial_{X}^{2} H_{2}+W^{\prime \prime}\left(H_{0}\right) H_{2}+W^{\prime \prime \prime}\left(H_{0}\right) \frac{H_{1}^{2}}{2}=v_{2} .
$$

Taking the limit $X \rightarrow+\infty$, we find

$$
v_{2}=-\bar{\gamma} \partial_{x}^{2} h_{1}\left(x_{\mathrm{TL}}\right)
$$

Similarly taking the $X \rightarrow-\infty$ limit, we obtain

$$
v_{2}=W^{\prime \prime}(0) \mathcal{H}_{1}\left(\chi_{\mathrm{TL}}\right)+W^{\prime \prime \prime}(0) \frac{\mathcal{H}_{0}^{2}\left(\chi_{\mathrm{TL}}\right)}{2} .
$$

To this order, we again obtain the equilibrium relations of no mass flux and equality of chemical potential on both sides of the triple line.

Now, from Eq. (A13) to second order we have

$$
\begin{aligned}
& {\left[U_{2}\right]_{-\infty}^{+\infty}-\tilde{v}_{0}(-\infty)\left[H_{2}\right]_{-\infty}^{+\infty}-\tilde{v}_{1}(-\infty)\left[H_{1}\right]_{-\infty}^{+\infty}} \\
& \quad-\tilde{v}_{2}(-\infty)\left[H_{0}\right]_{-\infty}^{+\infty}=0 .
\end{aligned}
$$

Using the matching conditions, we find that the diverging terms on both sides cancel. Thus, we obtain

$$
\begin{aligned}
& {\left[\tilde{U}_{2}\right]_{-\infty}^{+\infty}-\tilde{v}_{0}(-\infty)\left[\tilde{H}_{2}\right]_{-\infty}^{+\infty}-\tilde{v}_{1}(-\infty)\left[\tilde{H}_{1}\right]_{-\infty}^{+\infty}} \\
& \quad-\tilde{v}_{2}(-\infty)\left[\tilde{H}_{0}\right]_{-\infty}^{+\infty}=0
\end{aligned}
$$

which is the second-order contribution to the Young contact angle condition.

\section{d. Third order}

Consider Eq. (A8) to third order

$$
\begin{aligned}
-v_{0} \partial_{X} H_{0}= & \partial_{X}\left[B ( H _ { 0 } ) \partial _ { X } \left(-\bar{\gamma} \partial_{X}^{2} H_{3}+W^{\prime \prime}\left(H_{0}\right) H_{3}\right.\right. \\
& \left.\left.+W^{\prime \prime \prime}\left(H_{0}\right) H_{1} H_{2}+W^{\prime \prime \prime \prime}\left(H_{0}\right) \frac{H_{1}^{3}}{6}\right)\right] .
\end{aligned}
$$


Integrating this equation, we obtain

$$
\begin{aligned}
-v_{0} H_{0}-J_{3}= & B\left(H_{0}\right) \partial_{X}\left(-\bar{\gamma} \partial_{X}^{2} H_{3}+W^{\prime \prime}\left(H_{0}\right) H_{3}\right. \\
& \left.+W^{\prime \prime \prime}\left(H_{0}\right) H_{1} H_{2}+W^{\prime \prime \prime \prime}\left(H_{0}\right) \frac{H_{1}^{3}}{6}\right) .
\end{aligned}
$$

Now using the matching conditions in the limit $X \rightarrow-\infty$, we have

$$
J_{3}=-B(0) W^{\prime \prime}(0) \partial_{\chi} \mathcal{H}_{0}\left(\chi_{\mathrm{TL}}\right)=\mathcal{J}_{0}\left(\chi_{\mathrm{TL}}\right) .
$$

Similarly, in the limit of $X \rightarrow+\infty$, we obtain two equations

$$
\begin{gathered}
v_{0} \partial_{x} h_{0}\left(x_{\mathrm{TL}}\right)=\bar{\gamma} B(\infty) \partial_{x}^{4} h_{0}\left(x_{\mathrm{TL}}\right) \\
v_{0} h_{1}\left(x_{\mathrm{TL}}\right)+\mathcal{J}_{0}\left(\chi_{\mathrm{TL}}\right)=\bar{\gamma} B(\infty) \partial_{x}^{3} h_{1}\left(x_{\mathrm{TL}}\right)=\mathcal{J}_{0}\left(x_{\mathrm{TL}}\right)
\end{gathered}
$$

where the first equation arises from a constant contribution and the second equation comes from a contribution linear in $X$.

Integrating Eq. (A42), we find

$$
\begin{aligned}
& -v_{0} \int_{0}^{X} \frac{H_{0}^{\prime}}{B\left(H_{0}^{\prime}\right)} d X^{\prime}-\mathcal{J}_{0}\left(\chi_{\mathrm{TL}}\right) \int_{0}^{X} \frac{d X^{\prime}}{B\left(H_{0}^{\prime}\right)}+v_{3}(0) \\
& =-\bar{\gamma} \partial_{X}^{2} H_{3}+W^{\prime \prime}\left(H_{0}\right) H_{3}+W^{\prime \prime \prime}\left(H_{0}\right) H_{1} H_{2} \\
& \quad+W^{\prime \prime \prime \prime}\left(H_{0}\right) \frac{H_{1}^{3}}{6} .
\end{aligned}
$$

Taking the $X \rightarrow+\infty$ limit and using Eqs. (A44) and (A45) and matching conditions, we obtain

$$
\begin{aligned}
& -v_{0} \int_{0}^{+\infty}\left(\frac{H_{0}}{B\left(H_{0}\right)}-\frac{X \partial_{x} h_{0}\left(x_{\mathrm{TL}}\right)+h_{1}\left(x_{\mathrm{TL}}\right)}{B(\infty)}\right) d X \\
& -\mathcal{J}_{0}\left(\chi_{\mathrm{TL}}\right) \int_{0}^{+\infty} d X\left(\frac{1}{B\left(H_{0}\right)}-\frac{1}{B(\infty)}\right) \\
& =-\bar{\gamma} \partial_{x}^{2} h_{2}\left(x_{\mathrm{TL}}\right)-v_{3}(0) .
\end{aligned}
$$

Similarly, when $X \rightarrow-\infty$ and using Eq. (A43) and matching conditions, we have

$$
\begin{aligned}
& -v_{0} \int_{0}^{-\infty} \frac{H_{0}}{B\left(H_{0}\right)} d X-\mathcal{J}_{0}\left(\chi_{\mathrm{TL}}\right) \int_{0}^{-\infty} d X\left(\frac{1}{B\left(H_{0}\right)}-\frac{1}{B(0)}\right) \\
& =\left.W^{\prime}\left[\epsilon \mathcal{H}\left(\chi_{\mathrm{TL}}\right)\right]\right|_{3}-v_{3}(0),
\end{aligned}
$$

where

$$
\begin{aligned}
\left.W^{\prime}\left[\epsilon \mathcal{H}\left(\chi_{\mathrm{TL}}\right)\right]\right|_{3}= & W^{\prime \prime}(0) \mathcal{H}_{2}\left(\chi_{\mathrm{TL}}\right) \\
& +W^{\prime \prime \prime}(0) \mathcal{H}_{0}\left(\chi_{\mathrm{TL}}\right) \mathcal{H}_{1}\left(\chi_{\mathrm{TL}}\right) \\
& +\frac{W^{(4)}(0)}{6} \mathcal{H}_{0}^{3}\left(\chi_{\mathrm{TL}}\right) .
\end{aligned}
$$

Subtracting Eq. (A48) from Eq. (A47), we obtain a relation between the velocity and mass flux at the triple line, and the difference of chemical potential between the island and substrate regions

$$
-v_{0} L_{1 v}-\mathcal{J}_{0}\left(\chi_{\mathrm{TL}}\right) L_{1 J}=-\bar{\gamma} \partial_{x}^{2} h_{2}\left(x_{\mathrm{TL}}\right)-\left.W^{\prime}\left[\epsilon \mathcal{H}\left(\chi_{\mathrm{TL}}\right)\right]\right|_{3},
$$

where

$$
\begin{gathered}
L_{1 v}=\int_{-\infty}^{+\infty} d X\left(\frac{H_{0}}{B\left(H_{0}\right)}-\Theta(X) \frac{X \partial_{x} h_{0}\left(x_{\mathrm{TL}}\right)+h_{1}\left(x_{\mathrm{TL}}\right)}{B(\infty)}\right), \\
L_{1 J}=\int_{-\infty}^{+\infty} d X\left(\frac{1}{B\left(H_{0}\right)}-\frac{\Theta(X)}{B(\infty)}-\frac{\Theta(-X)}{B(0)}\right) .
\end{gathered}
$$

Similarly, from Eq. (A13), at $O\left(\epsilon^{3}\right)$, we have

$$
\begin{aligned}
& -v_{0} \int_{-\infty}^{+\infty} d X \partial_{X} H_{0} \int_{-\infty}^{X} d X^{\prime} \frac{H_{0}^{\prime}}{B\left(H_{0}^{\prime}\right)} \\
& -\mathcal{J}_{0}\left(\chi_{\mathrm{TL}}\right) \int_{-\infty}^{+\infty} d X \partial_{X} H_{0}\left(\int_{-\infty}^{X} \frac{d X^{\prime}}{B\left(H_{0}^{\prime}\right)}-\int_{-\infty}^{0} \frac{d X^{\prime}}{B(0)}\right) \\
& =\left[U_{3}\right]_{-\infty}^{+\infty}-\sum_{i=0}^{3} \tilde{v}_{i}(-\infty)\left[H_{3-i}\right]_{-\infty}^{+\infty}
\end{aligned}
$$

Again using the matching conditions and canceling the diverging terms, we obtain the relation to third order,

$$
-v_{0} \bar{L}_{2 v}-\mathcal{J}_{0}\left(\chi_{\mathrm{TL}}\right) L_{2 J}=\left[\tilde{U}_{3}\right]_{-\infty}^{+\infty}-\sum_{i=0}^{3} \tilde{v}_{i}(-\infty)\left[\tilde{H}_{3-i}\right]_{-\infty}^{+\infty},
$$

where $\bar{L}_{2 v}$ and $L_{2 J}$ are given by

$$
\begin{aligned}
\bar{L}_{2 v}= & \int_{-\infty}^{+\infty} d X\left[\partial_{X} H_{0} \int_{-\infty}^{X} d X^{\prime} \frac{H_{0}^{\prime}}{B\left(H_{0}^{\prime}\right)}-\frac{\Theta(X)}{B(\infty)}\right. \\
& \times\left(\frac{X^{2}}{2}\left(\partial_{x} h_{0}\right)^{2}\left(x_{\mathrm{TL}}\right)+X h_{1}\left(x_{\mathrm{TL}}\right) \partial_{x} h_{0}\left(x_{\mathrm{TL}}\right)\right) \\
& \left.-\Theta(X) \partial_{x} h_{0}\left(x_{\mathrm{TL}}\right) L_{1 v}\right], \\
L_{2 J}= & \int_{-\infty}^{+\infty} d X\left[\partial_{X} H_{0}\left(\int_{-\infty}^{X} \frac{d X^{\prime}}{B\left(H_{0}^{\prime}\right)}-\int_{-\infty}^{0} \frac{d X^{\prime}}{B(0)}\right)\right. \\
& \left.-\frac{\Theta(X) X \partial_{x} h_{0}\left(x_{\mathrm{TL}}\right)}{B(\infty)}-\Theta(X) \partial_{x} h_{0}\left(x_{\mathrm{TL}}\right) L_{1 J}\right] .
\end{aligned}
$$

\section{Kinetic boundary conditions}

Now, since $v_{0}, v_{1}$, and $v_{2}$ are constants, Eq. (A50) leads to the first kinetic boundary condition up to third order

$$
-\epsilon^{3} v_{0} L_{1 v}-\epsilon^{3} \mathcal{J}_{0}\left(\chi_{\mathrm{TL}}\right) L_{1 J}=[\tilde{v}]_{-\infty}^{+\infty} .
$$

Similarly, combining Eqs. (A25), (A31), (A40), and (A54) up to third order, we find

$$
-\epsilon^{3} v_{0} \bar{L}_{2 v}-\epsilon^{3} \mathcal{J}_{0}\left(\chi_{\mathrm{TL}}\right) L_{2 J}=[\tilde{U}]_{-\infty}^{+\infty}-\tilde{v}(-\infty)[\tilde{H}]_{-\infty}^{+\infty} .
$$

Finally, since the zeroth-, first-, and second-order contributions to the mass flux are zero, combining these contributions with 

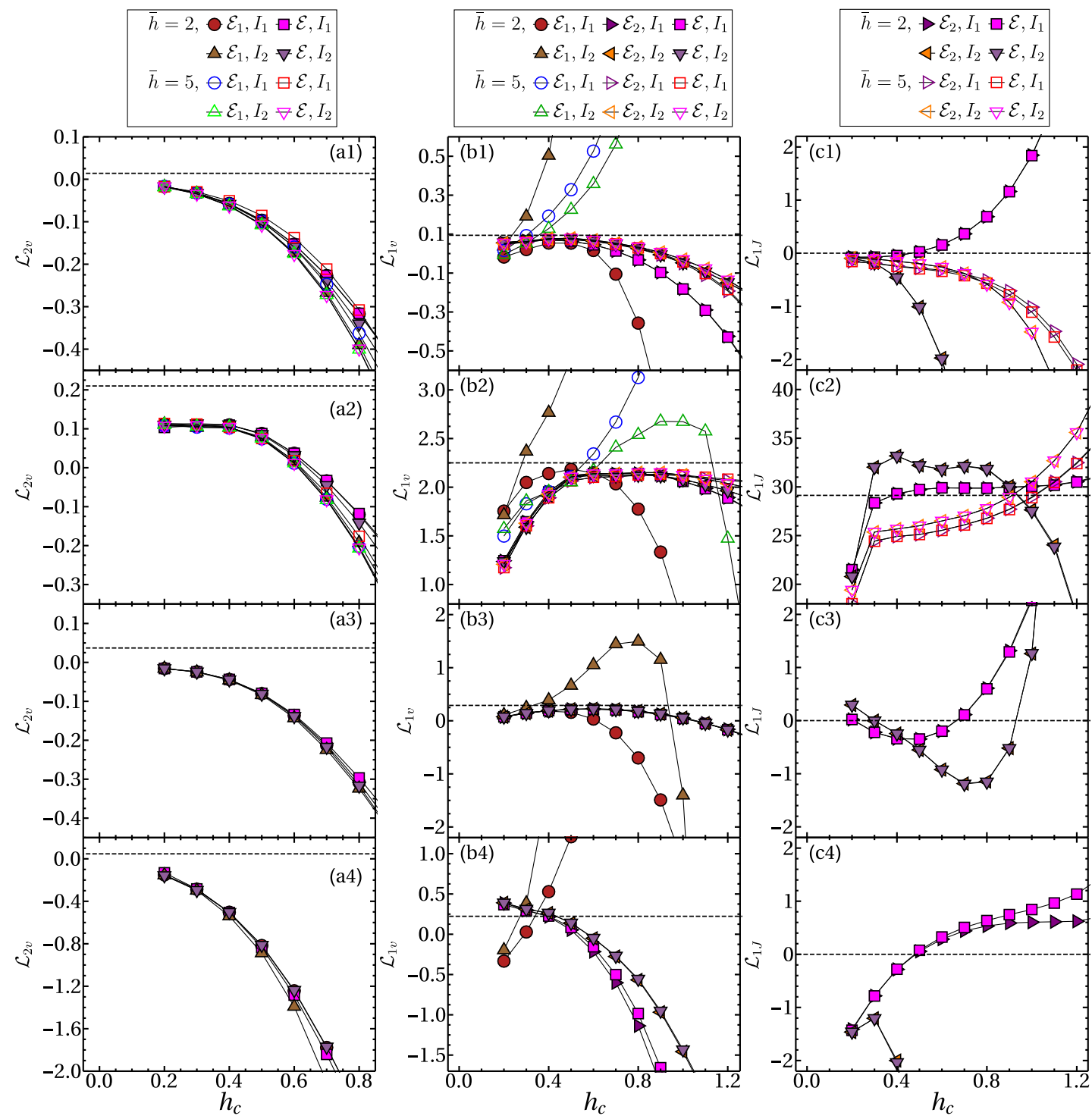

FIG. 11. Numerical verification of the kinetic boundary conditions. Here $j_{\mathrm{TL}}$ is the value obtained from the extrapolation of the mass flux from the island side. The notation and symbols are similar to those in Fig. 7.

Eq. (A45), we obtain the local mass conservation relation

$$
[\tilde{J}]_{-\infty}^{+\infty}=\epsilon^{3}\left[\mathcal{J}_{0}\left(x_{\mathrm{TL}}\right)-\mathcal{J}_{0}\left(\chi_{\mathrm{TL}}\right)\right]_{-\infty}^{+\infty}=\epsilon^{3} v_{0}\left[\tilde{H}_{0}\right]_{-\infty}^{+\infty} .
$$

In order to obtain relations between kinetic coefficients, we rewrite Eq. (A56) as

$$
\begin{aligned}
L_{2 J}= & \int_{-\infty}^{+\infty} d X\left[-\partial_{X} H_{0} \int_{X}^{+\infty} d X^{\prime}\left(\frac{1}{B\left(H_{0}^{\prime}\right)}-\frac{\Theta\left(X^{\prime}\right)}{B(\infty)}-\frac{\Theta\left(-X^{\prime}\right)}{B(0)}\right)+X \partial_{X} H_{0}\left(\frac{\Theta(-X)}{B(0)}+\frac{\Theta(X)}{B(\infty)}\right)\right. \\
& \left.-\frac{\Theta(X) X \partial_{x} h_{0}\left(x_{\mathrm{TL}}\right)}{B(\infty)}+\left[\partial_{X} H_{0}-\Theta(X) \partial_{x} h_{0}\left(x_{\mathrm{TL}}\right)\right] L_{1 J}\right] .
\end{aligned}
$$

Now integrating the first term on right-hand side and making further simplifications, we obtain Eq. (101). A similar rewriting of $\bar{L}_{2 v}$ leads to

$$
\begin{aligned}
\bar{L}_{2 v}= & \int_{-\infty}^{+\infty} d X\left[-\partial_{X} H_{0} \int_{X}^{+\infty} d X^{\prime}\left(\frac{H_{0}^{\prime}}{B\left(H_{0}^{\prime}\right)}-\frac{\Theta\left(X^{\prime}\right)}{B(\infty)}\left[X \partial_{x} h_{0}\left(x_{\mathrm{TL}}\right)+h_{1}\left(x_{\mathrm{TL}}\right)\right]\right)+\frac{\Theta(X)}{B(\infty)}\left[\partial_{X} H_{0}-\partial_{x} h_{0}\left(x_{\mathrm{TL}}\right)\right]\right. \\
& \left.\times\left(\frac{X^{2}}{2} \partial_{x} h_{0}\left(x_{\mathrm{TL}}\right)+X h_{1}\left(x_{\mathrm{TL}}\right)\right)-\left[\partial_{X} H_{0}-\Theta(X) \partial_{x} h_{0}\left(x_{\mathrm{TL}}\right)\right] L_{1 v}\right] .
\end{aligned}
$$

Again making some simplifications, we obtain Eq. (102). 
In order to write the kinetic boundary conditions in physical variables, we first relate physical variables with the corresponding variables in the triple-line region. These relations are defined as

$$
\begin{gathered}
j=\frac{J}{\epsilon^{2}}=\frac{J_{0}+\epsilon J_{1}+\epsilon^{2} J_{2}+\epsilon^{3} J_{3}+\cdots}{\epsilon^{2}}, \\
\mu=\frac{v}{\epsilon}=\frac{v_{0}+\epsilon v_{1}+\epsilon^{2} v_{2}+\epsilon^{3} v_{3}+\cdots}{\epsilon}, \\
u=U=U_{0}+\epsilon U_{1}+\epsilon^{2} U_{2}+\epsilon^{3} U_{3}+\cdots
\end{gathered}
$$

Using these relations, we may rewrite Eq. (A57) as

$$
-\epsilon^{3} v_{0} L_{1 v}-\epsilon^{3} J_{3} L_{1 J}=[\tilde{v}]_{-\infty}^{+\infty}
$$

leading to

$$
-\epsilon^{3} v L_{1 v}-\epsilon^{2} j_{-} L_{1 J}=\epsilon[\mu]_{\mathrm{TL}} .
$$

Then, simplifying by $\epsilon$, we find

$$
-v\left(\epsilon^{2} L_{1 v}\right)-j_{-}\left(\epsilon L_{1 J}\right)=[\mu]_{\mathrm{TL}},
$$

which may be written as

$$
-v \mathcal{L}_{1 v}-j_{-} \mathcal{L}_{1 J}=[\mu]_{\mathrm{TL}}
$$

with $\mathcal{L}_{1 v}=\epsilon^{2} L_{1 v}$ and $\mathcal{L}_{1 J}=\epsilon L_{1 J}$. Following the same lines, we rewrite Eq. (A58) as

$$
-\epsilon^{3} v_{0} \bar{L}_{2 v}-\epsilon^{3} J_{3} L_{2 J}=[\tilde{U}]_{-\infty}^{+\infty}-\tilde{v}(-\infty)[\tilde{H}]_{-\infty}^{+\infty},
$$

leading to

$$
-\epsilon^{3} v \bar{L}_{2 v}-\epsilon^{2} j_{-} L_{2 J}=[u]_{\mathrm{TL}}-\epsilon \tilde{\mu}_{-} \frac{[h]_{\mathrm{TL}}}{\epsilon},
$$

which is rewritten as

$$
-v \overline{\mathcal{L}}_{2 v}-j_{-} \mathcal{L}_{2 J}=[u]_{\mathrm{TL}}-\tilde{\mu}_{-}[h]_{\mathrm{TL}},
$$

with $\overline{\mathcal{L}}_{2 v}=\epsilon^{3} \bar{L}_{2 v}$ and $\mathcal{L}_{2 J}=\epsilon^{2} L_{2 J}$. Finally, the relation (A59) can be written

$$
\epsilon^{2}[j]_{\mathrm{TL}}=\epsilon^{2} v[h]_{\mathrm{TL}} .
$$

Simplifying by $\epsilon^{2}$, we find

$$
[j]_{\mathrm{TL}}=v[h]_{\mathrm{TL}} .
$$

Multiplying Eq. (A68) by $[h]_{\mathrm{TL}}$ and subtracting from Eq. (A71), we obtain the kinetic boundary conditions (90) and (91) of the main text.

\section{APPENDIX B: SIMULATION RESULTS FOR THE CONSERVED CASE WITH FLUXES FROM THE FILM SIDE}

Here we show Fig. 11, which summarizes the numerical evaluation of the kinetic coefficients in the conserved case using the mass flux calculated from the film side.
[1] P.-G. de Gennes, F. Brochard-Wyart, and D. Quere, Capillarity and Wetting Phenomena: Drops, Bubbles, Pearls, Waves (Springer, New York, 2013).

[2] D. Bonn, J. Eggers, J. Indekeu, J. Meunier, and E. Rolley, Rev. Mod. Phys. 81, 739 (2009).

[3] C. Huh and L. E. Scriven, J. Colloid Interface Sci. 35, 85 (1971).

[4] M. Rivetti, T. Salez, M. Benzaquen, and E. Raphaël, Soft Matter 11, 9247 (2015).

[5] Y. Deng, L. Chen, Q. Liu, J. Yu, and H. Wang, J. Phys. Chem. Lett. 7, 1763 (2016).

[6] R. Ledesma-Aguilar, A. Hernández-Machado, and I. Pagonabarraga, Phys. Rev. Lett. 110, 264502 (2013).

[7] H. Kusumaatmaja, E. J. Hemingway, and S. M. Fielding, J. Fluid Mech. 788, 209 (2016).

[8] U. Czubayko, V. Sursaeva, G. Gottstein, and L. Shvindlerman, Acta Mater. 46, 5863 (1998).

[9] M. Upmanyu, D. Srolovitz, L. Shvindlerman, and G. Gottstein, Acta Mater. 50, 1405 (2002).

[10] D. J. Srolovitz and S. A. Safran, J. Appl. Phys 60, 247 (1986).

[11] H. Wong, P. Voorhees, M. Miksis, and S. Davis, Acta Mater. 48, 1719 (2000).

[12] G. H. Kim, R. V. Zucker, J. Ye, W. C. Carter, and C. V. Thompson, J. Appl. Phys. 113, 043512 (2013).

[13] M. Salvalaglio, R. Backofen, R. Bergamaschini, F. Montalenti, and A. Voigt, Cryst. Growth Design 15, 2787 (2015).

[14] O. Pierre-Louis, A. Chame, and Y. Saito, Phys. Rev. Lett. 103, 195501 (2009).
[15] M. Ignacio, Y. Saito, P. Smereka, and O. Pierre-Louis, Phys. Rev. Lett. 112, 146102 (2014).

[16] Y. Wang, W. Jiang, W. Bao, and D. J. Srolovitz, Phys. Rev. B 91, 045303 (2015)

[17] W. W. Mullins, J. Appl. Phys. 28, 333 (1957).

[18] J. Becker, G. Grün, R. Seemann, H. Mantz, K. Jacobs, K. R. Mecke, and R. Blossey, Nat. Mater. 2, 59 (2003).

[19] L. M. Pismen and U. Thiele, Phys. Fluids 18, 042104 (2006).

[20] U. Thiele, J. Phys.: Condens. Matter 22, 084019 (2010).

[21] A. Alizadeh Pahlavan, L. Cueto-Felgueroso, G. H. McKinley, and R. Juanes, Phys. Rev. Lett. 115, 034502 (2015).

[22] O. Pierre-Louis, Prog. Cryst. Growth Charact. Mater. 62, 177 (2016).

[23] A. A. Golovin, M. S. Levine, T. V. Savina, and S. H. Davis, Phys. Rev. B 70, 235342 (2004).

[24] J.-N. Aqua and T. Frisch, Phys. Rev. B 82, 085322 (2010).

[25] M. Khenner, Phys. Rev. B 77, 165414 (2008).

[26] A. Constantinescu, L. Golubović, and A. Levandovsky, Phys. Rev. E 88, 032113 (2013).

[27] G. Caginalp, Phys. Rev. A 39, 5887 (1989).

[28] O. Pierre-Louis, Phys. Rev. E 68, 021604 (2003).

[29] C. V. Thompson, Annu. Rev. Mater. Res. 42, 399 (2012).

[30] A. Chame and O. Pierre-Louis, C. R. Phys. 14, 553 (2013).

[31] D. T. Danielson, D. K. Sparacin, J. Michel, and L. C. Kimerling, J. Appl. Phys. 100, 083507 (2006).

[32] E. Dornel, J.-C. Barbé, F. de Crécy, G. Lacolle, and J. Eymery, Phys. Rev. B 73, 115427 (2006). 
[33] E. Bussmann, F. Cheynis, F. Leroy, and P. Müller, IOP Conf. Ser.: Mater. Sci. Eng. 12, 012016 (2010).

[34] E. Bussmann, F. Cheynis, F. Leroy, P. Müller, and O. PierreLouis, New J. Phys. 13, 043017 (2011).

[35] F. Leroy, F. Cheynis, T. Passanante, and P. Müller, Phys. Rev. B 85, 195414 (2012).

[36] F. Cheynis, F. Leroy, T. Passanante, and P. Müller, Appl. Phys. Lett. 102, 161603 (2013).

[37] J. Ye and C. V. Thompson, Appl. Phys. Lett. 97, 071904 (2010); Phys. Rev. B 82, 193408 (2010); Acta Mater. 59, 582 (2011).

[38] E. Jiran and C. V. Thompson, J. Electron. Mater. 19, 1153 (1990); Thin Solid Films 208, 23 (1992).

[39] C. Kennefick and R. Raj, Acta Metal. 37, 2947 (1989).

[40] G.-H. Lu and F. Liu, Phys. Rev. Lett. 94, 176103 (2005).

[41] J. Israelachvili, Intermolecular and Surface Forces (Elsevier Science, Amsterdam, 2015).

[42] The singular limiting case where the substrate mobility vanishes at the substrate $M(H) \rightarrow 0$ as $H \rightarrow 0$ corresponds to the absence of relaxation on the substrate. It is not clear whether this limit can be caught by our expansion. However, if we assume $M(H) \rightarrow H^{\alpha}$ as $H \rightarrow 0$, the convergence of $\mathcal{L}_{v}$ requires that $\alpha<2$.

[43] Y. Han and D.-J. Liu, Phys. Rev. B 80, 155404 (2009).
[44] B. Wu and Z. Zhang, Phys. Rev. B 77, 035410 (2008).

[45] H. Hirayama, Surf. Sci. 603, 1492 (2009).

[46] J. D. Jackson, Classical Electrodynamics (Wiley, New York, 1998).

[47] C. Canuto, M. Y. Hussaini, A. M. Quarteroni, and T. A. Zang, Jr., Spectral Methods in Fluid Dynamics (Springer, Berlin, 1987).

[48] M. C. Cross, D. Meiron, and Y. Tu, Chaos 4, 607 (1994).

[49] W. Press, Numerical Recipes 3rd Edition: The Art of Scientific Computing (Cambridge University Press, Cambridge, 2007).

[50] M. Frigo and S. G. Johnson, Proc. IEEE 93, 216 (2005), special issue on program generation, optimization, and platform adaptation.

[51] S. Cox and P. Matthews, J. Comput. Phys. 176, 430 (2002).

[52] A. Chame and O. Pierre-Louis, Phys. Rev. E 85, 011602 (2012).

[53] F. Cheynis, E. Bussmann, F. Leroy, T. Passanante, and P. Müller, Phys. Rev. B 84, 245439 (2011).

[54] M. Naffouti, R. Backofen, M. Salvalaglio, T. Bottein, M. Lodari, A. Voigt, T. David, A. Benkouider, I. Fraj, L. Favre, A. Ronda, I. Berbezier, D. Grosso, M. Abbarchi, and M. Bollani, Sci. Adv. 3, eaao1472 (2017).

[55] C. Misbah, O. Pierre-Louis, and Y. Saito, Rev. Mod. Phys. 82, 981 (2010).

[56] M. Napiórkowski and S. Dietrich, Phys. Rev. E 47, 1836 (1993). 